\title{
Biomedical Papers
}

Formerly ACTA

UNIVERSITATIS PALACKIANAE OLOMUCENSIS

FACULTATIS MEDICAE, Volumes 1-144

VOLUME 162, SUPPLEMENT 1 JUNE 2018

$5^{\text {th }}$ Central-Eastern European Congress

on Cell-Free Nucleic Acids

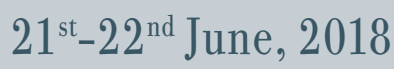

Guest Editor:

Prof. Balint Nagy, Debrecen, Hungary

PALACKÝ UNIVERSITY OLOMOUG 


\title{
BIOMEDICAL PAPERS (Biomed Pap Med Fac Univ Palacky Olomouc Czech Repub)
}

(formerly Acta Universitatis Palackianae Olomucensis Facultatis Medicae, Volumes 1-144)

EDITOR-IN-CHIEF

Jitka Ulrichová, Olomouc, Czech Republic
CO-EDITOR-IN-CHIEF

Vilím Šimánek, Olomouc, Czech Republic

\author{
ASSOCIATED EDITORS \\ Jan Bouchal, Olomouc, Czech Republic \\ Jan Václavík, Olomouc, Czech Republic
}

\section{EDITORIAL BOARD}

Petr Bachleda, Olomouc, Czech Republic Július Brtko, Bratislava, Slovak Republic Tomáš Eckschlager, Praha, Czech Republic Miroslav Heřman, Olomouc, Czech Republic Maurizio Iacobone, Padova, Italy Vojtěch Kamarád, Olomouc, Czech Republic Jan Kováč, Leicester, United Kingdom Evžen Křepela, Praha, Czech Republic Arnošt Martínek, Ostrava, Czech Republic Bohuslav Melichar, Olomouc, Czech Republic Paul Gerard Murray, Birmingham, United Kingdom Ivan Netuka, Praha, Czech Republic Vladimír Palička, Hradec Králové, Czech Republic Michal Rygl, Praha, Czech Republic Martin Smrčka, Brno, Czech Republic David Stejskal, Prostejov, Czech Republic Jan Václavík, Olomouc, Czech Republic Jiří Vítovec, Brno, Czech Republic Zdeněk Zadák, Hradec Králové, Czech Republic Josef Zadražil, Olomouc, Czech Republic David Zeman, Ostrava, Czech Republic

\section{AIMS AND SCOPE}

Biomedical Papers is an official journal of the Palacký University, Faculty of Medicine and Dentistry, Olomouc, Czech Republic which publishes reviews and original papers relevant to all biomedical disciplines. The journal publishes one volume per year consisting of four issues.

\section{INDEXING}

This journal is indexed in BIBLIOGRAPHIA MEDICA CECHOSLOVACA, CHEMICAL ABSTRACTS, GOOGLE SCHOLAR, INDEX MEDICUS/MEDLINE, ISI WEB OF SCIENCE, and SCOPUS

\section{All correspondence should be addressed to:}

Jana Nováková, Department of Medical Chemistry and Biochemistry (BIOMEDICAL PAPERS) Faculty of Medicine and Dentistry, Palacký University, Hněvotínská 3, 77515 Olomouc, Czech Republic. E-mail: biomed.papers@upol.cz, Tel/Fax: +420 585632302 


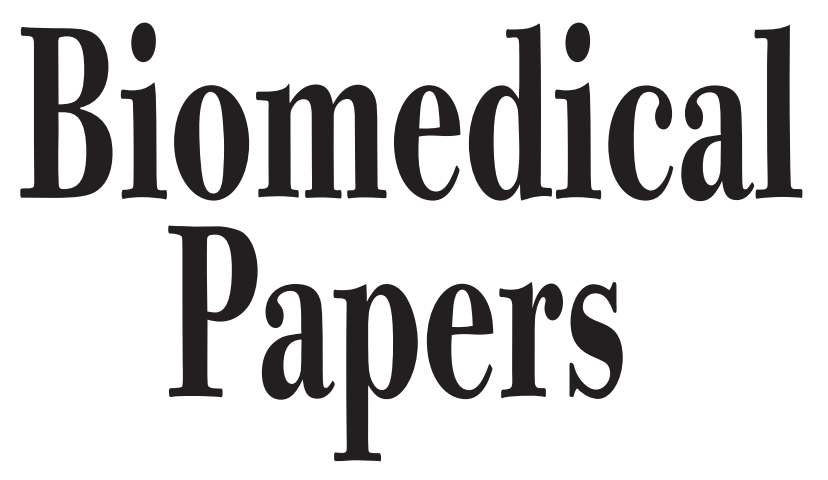

VOLUME 162, SUPPLEMENT 1

PALACKÝ UNIVERSITY OLOMOUG

2018 


\section{BIOMEDICAL PAPERS}

Volume 162, Supplement 1

$$
\begin{aligned}
& \text { Published quarterly } \\
& \text { MK ČR E } 12793
\end{aligned}
$$

Published by Palacký University Olomouc Křížkovského 8, 77147 Olomouc, IČO 61989592

Olomouc 2018

eISSN 1804-7521

http://dx.doi.org/10.5507/bp.2018.029 


\section{$5^{\text {th }}$ Central-Eastern European Congress on Cell-Free Nucleic Acids $21^{\text {st }}-22^{\text {nd }}$ June, 2018}

Organized by the

Department of Human Genetics

Faculty of Medicine

University of Debrecen, Debrecen, Hungary 


\section{Organizer}

Department of Human Genetics, Faculty of Medicine, University of Debrecen, Debrecen, Hungary

\section{Invited Speakers}

Folkert W. Asselbergs (University Medical Center, Utrecht)

Howard S. Cuckle (University of Tel Aviv, Tel Aviv)

Christoph Sensen (Graz University, Graz)

Ripudaman Singh (ARCEDI Biotech ApS, Vejle, Denmark)

Erik A. Sistermans (VU University Medical Center, Amsterdam)

\section{International Scientific Committee}

Peter Celec (Comenius University, Bratislava)

Ilona Hromadnikova (Charles University, Prague)

Zora Lasabova (Comenius University, Martin)

Tomas Szemes (Comenius University, Bratislava)

Jasenka Wagner (J.J. Strossmayer University, Osijek)

\section{Local Organizing Committee}

Balint Nagy (chairman, University of Debrecen, Debrecen)

Zoltan Csanadi (vice-chairman, University of Debrecen, Debrecen)

Gabor Mehes (vice-chairman, University of Debrecen, Debrecen)

Robert Poka (vice-chairman, University of Debrecen, Debrecen)

Peter Igaz (Semmelweis University, Budapest)

Maria Judit Molnar (Semmelweis University, Budapest)

Sandor Nagy (Petz A. County Hospital, Gyor)

Beata Soltesz (University of Debrecen, Debrecen)

Olga Torok (University of Debrecen, Debrecen) 


\section{Invitation}

On behalf of the Department of Human Genetics of the Faculty of Medicine, University of Debrecen it is my pleasure to welcome you to the $5^{\text {th }}$ Central-Eastern European Congress on Cell-Free Nucleic Acids in Debrecen.

Recent advances in cell-free nucleic acid research have opened up new prospects for their introduction into the clinical practice. Application of cell-freeDNA in the prenatal detection of genetic diseases has already become a routine procedure in non-invasive prenatal testing (NIPT). Now there is a growing interest in using cell-free nucleic acids (cfNA) obtained from liquid biopsy in the field of oncology, and also in the diagnosis/treatment of cardiovascular diseases. The role of microvesicle encapsulation of the cfNAs has also arisen as an exciting new area of research, where the biological role these particles play in the preservation of homeostasis and in the development of diseases is still unknown. Furthermore, there is also the possibility of horizontal gene transfer (genometastasis), which has also piqued the curiosity of scientists.

The first Central-Eastern European Congress on Cell Free Nucleic Acids was held in Budapest in 2010, and has since been held biennially: in Olomouc (2012), Martin (2014), and in Split (2016).

The previous conferences' topics have mainly been on the research and application of cfNAs in the prenatal diagnosis, however, this year we wish to extend it to their application in oncological and cardiovascular diseases. We have invited several world famous scientists in these fields as speakers to share their knowledge with Central European researchers and clinicians.

We look forward to giving you the unforgettable memory of an enlightening congress, and to giving you the warm welcome of the people and city of Debrecen.

Balint Nagy

Chairman of the Organizing Committee 


\title{
CONTENTS
}

\author{
Invited Speakers (IS) ...................................... 1 \\ Lectures (L) ...................................................... 3

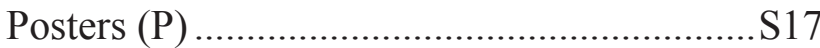 \\ Presentations of Companies (CP) ..................S20
}


IS-1

\section{Comparing cfDNA tests for aneuploidy}

\section{Howard Cuckle}

Department of Obstetrics and Gynecology, Tel Aviv University, Tel Aviv, Israel; Department of Obstetrics and Gynecology, Columbia University Medical Center, New York, NY, USA; Reproductive Epidemiology, University of Leeds, Leeds, UK

It is now established beyond doubt that cell-free (cf) DNA testing in early pregnancy has superior screening performance than conventional screening strategies. The Down syndrome detection rate exceeds $99 \%$ and the falsepositive rate is under $0.5 \%$. By comparison the widely used conventional Combined test has a model predicted detection rate of $84 \%$ for a false-positive rate of $5 \%$. And even the best available conventional approaches that include additional ultrasound and biochemical markers do not have a predicted detection rate over $95 \%$ for a falsepositive rate of $5 \%$.

However, the choice of which commercial or in-house cfDNA to offer patients is not straight-forward.

The tests will differ according to their test failure or 'no-call' rates. And the extent to which this matters will be dependent on whether cfDNA testing is offered as a secondary test after a positive conventional screening test result, as a contingent test on $20-30 \%$ of women selected for testing by the conventional test or as a primary screening test.

Furthermore, the information needed to make a fair comparison between cfDNA test providers is not available. A simultaneous comparison in detection rate, falsepositive rate and no-call rate is needed. This would ideally be done by 'back-to-back' testing of the same panel of samples but to my knowledge only one such study has been published and it is underpowered to determine statistically significant differences.

\section{IS-2}

\section{Introducing NIPT analysis in the Netherlands part II, from TRIDENT-1 to TRIDENT-2}

\section{Erik A. Sistermans}

\section{VU University Medical Center Amsterdam, the Netherlands}

The Netherlands are the first country where NIPT is incorporated into a governmentally supported and health care funded prenatal Down syndrome screening program. In many countries, NIPT has been introduced commercially, without governmental guidance. In the Netherlands the Population Screening Act regulates the introduction of screening programs for untreatable diseases such as Down syndrome. April $1^{\text {st }} 2014$, the Dutch NIPT consortium, consisting of all relevant stakeholders, obtained a license for 2 years for a nationwide NIPT implementation study for pregnant women at increased risk of having an affected child, called TRIDENT (Trial by Dutch laboratories for evaluation of non-invasive prenatal testing). Inclusion criteria are a risk of $>1: 200$ for trisomy (T) 21 , 18 or 13 based on the first trimester combined test, or medical history. This study, now known as TRIDENT-1, has resulted in three major and several more minor publications, which will be summarized during the presentation. This will include data on full clinical follow up, and information on findings other than trisomy 21, 13 or 18 .

A second license was obtained in September 2016 for a follow-up study called TRIDENT-2, where we could offer NIPT to all pregnant women starting April $1^{\text {st }} 2017$. This meant that we had only six months to set up this nationwide scheme, and to increase our labflow from 3000 samples to 70.000 samples a year. Furthermore, we needed to educate 3000 counsellors, had to set up a logistic scheme for all blood tubes, an ordering and reporting database etcetera. An overview of the way we managed to solve this huge challenge will be presented.

IS-3

\section{Invasive and non-invasive prenatal detection of micro-deletions}

\section{Howard Cuckle}

Department of Obstetrics and Gynecology, Tel Aviv University, Tel Aviv, Israel; Department of Obstetrics and Gynecology, Columbia University Medical Center, New York, NY, USA; Reproductive Epidemiology, University of Leeds, Leeds, UK

It is cost-effective for women with positive conventional screening tests for aneuploidy to be offered a choice between cell-free (cf)DNA or invasive prenatal diagnosis. There have now been a number of studies where those at high risk of aneuploidy, whether following screening, because of family history or other indication, have been given this choice. The results are not consistent between studies with the majority opting one way or the other.

In these circumstances, a fully informed choice would need to include information on the predictive value for micro-deletion syndromes using the cfDNA and the invasive tests that are being provided. Many cfDNA tests now incorporate five clinically important and relatively common micro-deletion syndromes: $1 \mathrm{p} 36$ deletion; cridu-chat (5p14-15 deletion); Prader-Willi and Angelman (15q11.2 paternal and maternal deletion, respectively); and the 22q11.2 deletion. Moreover, some but not all, invasive tests will incorporate a micro-array which can detect most copy-number variants, a proportion of which are associated with syndromes although many are benign or of unknown clinical significance.

As the cost of cfDNA testing falls the choice may be offered to even larger numbers of women.

In this presentation, the literature on the subject is reviewed and modeling is carried out with the aim of providing the information needed to inform choice in all such situations. 


\section{IS-4}

Fetal cells in maternal blood for prenatal diagnosis - the story so far

\section{Ripudaman Singh ${ }^{\mathrm{a}, \mathrm{b}}$, Lotte Hatt ${ }^{\mathrm{a}}$, Inga Baasch Christesen ${ }^{\mathrm{a}}$, Katarina Ravn ${ }^{\mathrm{a}}$, Olav Bjorn Petersen ${ }^{c, e}$, Niels Uldbjerg ${ }^{c}$, Rikke Christensen ${ }^{\mathrm{d}}$, Ida Vogel ${ }^{\mathrm{d}, \mathrm{e}}$, Palle Schelde ${ }^{\mathrm{a}}$}

\section{${ }^{a}$ ARCEDI Biotech ApS, Vejle, Denmark \\ ${ }^{b}$ Aarhus University, Aarhus, Denmark \\ 'Department of Obstetrics and Gynecology, Aarhus University Hospital, Aarhus, Denmark \\ ${ }^{d}$ Department of Clinical Genetics, Aarhus University Hospital, Aarhus, Denmark \\ eCentre for Prenatal Diagnostics, Aarhus University Hospital, Aarhus, Denmark \\ Correspondence to: rs@arcedi.com}

Few cells from the developing fetus invade the maternal circulation and this process starts as early as in the first weeks of the pregnancy. For more than half a century attempts have been made to isolate and use these very rare cells for prenatal testing, but with limited success. Technological advances in enrichment, manipulation and analyses of rare fetal cells from maternal blood made in the last 5 years have invigorated interest in circulating fetal cells and their application in prenatal testing. The hope is that enriched fetal cells from maternal blood can be used as superior alternatives to conventional NIPT which is based on free fetal DNA in maternal blood. For the last 11 years we have tried to answer some critical questions pertaining to fetal cells and their use in prenatal diagnosis. Studies have been done where the genetic results acquired from fetal cells were compared with genetic results after chorionic villi sampling. After developing a robust technology which is both scalable and cost effective, ARCEDI Biotech, in collaboration with Aarhus University Hospital has launched the first ever cell based NIPD in the central region of Denmark, covering approximately 17,000 pregnancies per year.

\section{IS-5}

\section{Cardiomyopathies: A genetic and phenotypic heterogeneous group of diseases}

\section{Folkert W. Asselbergs}

\section{Department of Cardiology, Division Heart \& Lungs University Medical Center Utrecht, the Netherlands \\ Correspondence to: f.w.asselbergs@umcutrecht.nl}

Cardiomyopathies are disorders of cardiac muscle with very high morbidity and mortality leading to chronic end-stage heart failure or sudden cardiac death. Mutations in over 60 genes encoding crucial structures of cardiomyocytes, such as sarcomeric filaments, calcium-metabolizing proteins, desmosomes, or mitochondrial enzymes, are causing cardiomyopathies. These discoveries have led to new recommendations for diagnostic evaluation to find the genetic cause of patient's disease and to predict disease prognosis. Next to the screening of the index patient, genetic counseling and segregation analysis is offered to first-degree family members for an early recognition of individuals at risk. However, a large portion of family members, who turn out to be pathogenic mutation carriers after the cascade screening, do not develop cardiomyopathy throughout life, which makes phenotype-genotype correlations in clinics unpredictable. This intrafamilial variability represents a significant social, economic and medical problem as asymptomatic mutation carriers may undergo unnecessary cardiologic testing, treatment with drugs that cause negative side-effects or implantation of cardiac devices that will never fire. On the other hand, mutation carriers at risk, who are not identified properly, may be undertreated and die suddenly at a young age. As the number of newly identified mutations in genes causing cardiomyopathies in patients and their family members is still rising, there is an urgent need to improve risk stratification in healthy individuals carrying a mutation and to design therapies to prevent and/or delay onset of cardiomyopathy.

IS-6

\section{Circulating nucleic acids as early markers for infectious and chronic diseases}

\section{Christoph W. Sensen}

\section{Institute of Computational Biotechnology, Graz University of Technology, Graz, Austria}

We are using serum-based circulating nucleic acids to identify markers, which are informative of early stages of infectious and chronic diseases in humans, production animals and pets, respectively. Usually, time-series studies of patients and controls are conducted, where DNA is extracted from serum samples (4-5 time points per individual), randomly amplified and then subjected to paired-end high-throughput DNA sequencing (Illumina). The resulting reads are mapped to the host genome and analysed with a dedicated Bioinformatics pipeline, which is capable of identifying DNA patterns, which are more or less prominent in patients with respect to internal standards (i.e. markers, which do not vary in patients and controls, respectively). Using the marker sets, we are attempting to develop RT-PCR assays, which are especially informative for early disease stages. 
$\mathrm{L}-1$

\section{Decreasing the no call rate of Non-Invasive Prenatal Testing}

\section{Marian Grendar ${ }^{\mathrm{a}}$, Dusan Loderer ${ }^{\mathrm{b}, \mathrm{c}}$, Iveta Svecova ${ }^{\mathrm{b}}$, Zuzana Laucekovab ${ }^{b}$ Michaela Hrtankovab ${ }^{b}$, Andrea Hornakovac, Balint Nagy ${ }^{d}$, Zora Lasabova ${ }^{e}$, Jan Danko ${ }^{b}$}

\author{
${ }^{a}$ Bioinformatic unit, Biomedical Center Martin, Jessenius Faculty \\ of Medicine, Comenius University, Martin, Slovak Republic \\ ${ }^{b}$ Department of Obstetrics and Gynecology, Jessenius Faculty of \\ Medicine, Comenius University, Martin, Slovak Republic \\ 'Division of Molecular Biology, Biomedical Center Martin, \\ Jessenius Faculty of Medicine, Comenius University, Martin, \\ Slovak Republic \\ ${ }^{d}$ Department of Human Genetics, Faculty of Medicine, University \\ of Debrecen, Debrecen, Hungary \\ ${ }^{e}$ Division of Oncology, Biomedical Center Martin, Jessenius \\ Faculty of Medicine, Comenius University, Martin, Slovak \\ Republic \\ Correspondence to: marian.grendar@jfmed.uniba.sk
}

The no call (or failure) rate is an important characteristics of any diagnostic, testing, or screening method. Failures of the read counting approach to Non-Invasive Prenatal Testing (NIPT) are primarily caused by the low fetal fraction. If fetal fraction is below a value, known as the Limit of Detection (LoD), then NIPT cannot determine the aneuploidy status of a fetus. The LoD can be estimated experimentally or by the coverage method. The coverage method implies that the LoD, and hence also the no call rate due to the low fetal fraction, can be made arbitrarily small by increasing the sequencing depth. The no call rate can be made arbitrarily small also by excluding from NIPT the pregnancies suspect of having fetal fraction below the LoD (e.g., due to the low gestational age, high maternal BMI, etc.). These methods of decreasing the no call rate have obvious drawbacks.

Explorations of the quantitative foundations of NIPT lead a variant of NIPT that for a fixed sequencing depth attains the no call rate lower than the conventional NIPT and is particularly useful for the subpopulation of pregnancies with the high maternal BMI, and/or low gestational week.
$\mathrm{L}-2$

\section{PANORAMA ${ }^{\oplus}$ the SNP sequencing-based NIPT - Hungarian results in the light of American data}

\author{
Balint Tobias ${ }^{\mathrm{a}}$, Lili Keszne Fodor ${ }^{\mathrm{a}}$, Sarolta Szoke ${ }^{\mathrm{a}}$, \\ Gabor Molnar ${ }^{a}$, Valeria Klujber, Bernadett Balla ${ }^{a}$, \\ Kovesdi Andrea ${ }^{\mathrm{a}}$, Peter Lakatos ${ }^{\mathrm{a}, \mathrm{b}}$, Janos P. Kosa ${ }^{\mathrm{a}, \mathrm{b}}$
}

aPentaCore Laboratory, Budapest, Hungary

ㄴ. Department of Internal Medicine Budapest, Semmelweis

University, Hungary

Introduction. Prenatal care demands a personalized and multidisciplinary approach. Ultrasound is an integral part of prenatal care but may also be complemented with further blood tests. The non-invasive prenatal screening tests (NIPTs) are already and elemental section of such nursing. The SNP (single nucleotide polymorphism)based identification of cell-free fetal DNA represent the newest generation of NIP tests, that overtake Massive Parallel Sequencing (MPS)-based tests both in sensitivity and specificity, also having a two-times higher PPV (positive predictive value) compared to the other so-called counting methods.

Methods. Panorama NIP screening test is available in Hungary from May 2015. The near hundred thousand population-based American follow-up study was taken in account when analysing the first 1000 Panorama test results from Hungary.

Results. NIPTs are currently used as second- or thirdline of prenatal screening tests, however their sensitivity and specificity exceeds $99 \%$. The newest generation of Panorama test has been carried out more than one million times, is based on the SNP sequencing of cell-free fetal DNA. Based on international data the combined PPV for trisomies 21 and 18 are $96.8 \%$ of Panorama, compared to the MPS-based tests' $43.8 \%$. Furthermore, Panorama has lower false-positive $(0.21 \%$ vs. $0.03 \%)$ and false-negative $(1.5 \%$ vs. $0.67 \%)$ rates. Furthermore, the SNP sequencing-based technology allows for the identification of fetal triploidy, vanishing twin, mosaicism, and hence the significant decrease in „no call” rates. The extreme high sensitivity (>99\%) and specificity (>99.9\%) rates of Panorama ${ }^{\circledR}$ also result from the technology. The most current national data are under analysis, which will be presented at the congress.

Conclusion. NIP tests are indispensable besides ultrasonography in the high-standard prenatal care. It is important to mention that NIP tests are screening tests, where high risk results must be verified by diagnostic tests. Furthermore, a uniform professional consensus must be brought. 
L-3

\section{First trimester screening for Down's syndrome by combined nuchal transluceny and prenatal cf-DNA testing}

\author{
Darija Strah ${ }^{a}$, Nina Ruzic Gorenjec ${ }^{b}$
}

\section{aStrah Diagnostic Centre, Domzale, Slovenia 'Institute for Biostatistics and Medical Informatics, Faculty of Medicine, University of Ljubljana, Ljubljana, Slovenia Correspondence to: darija@strah.si}

Objectives. The use of prenatal cf-DNA testing as a highly accurate screening method for aneuploidies is bringing radical changes in the field of fetal medicine. It results in decline of the number of invasive procedures in women with high risk (HR) based on maternal age and/ or nuchal translucency (NT) screening due to substitution with cf-DNA testing. The average age of women deciding to have cf-DNA testing has been similar in the last 4 years. However, the percentage of women with cf-DNA testing is increasing also in the low risk group due to high reassurance.

Methods. We present the results of prenatal cf-DNA testing in the period from 1. 1.2013 until 30. 6. 2017 in a private outpatient clinic. In our study, all of 9426 pregnant women had NT screening. Among them, 388 had also prenatal cf-DNA testing.

Results. The number of women having NT screening and adding cf-DNA testing has increased over the last 4 years (2013: 39, 2014: 70, 2015: 48, 2016: 157).The percentage of women with cf-DNA testing among the ones with NT screening raised from $1.45 \%$ in 2013 to $9.13 \%$ in 2016 (2014: 2.85\%, 2015: 2.74\%). For women that are HR only due to their age (37 years or more), this percentage increased from $13.38 \%$ in 2013 to $44.03 \%$ in 2016. Among women that are HR for T21 due to both age and prior NT screening, $5.65 \%$ decided to undergo cf-DNA testing in 2013 and $46.30 \%$ in 2016. In 2016, 4.24\% of women younger than 37 years decided for cf-DNA testing regardless of low risk NT screening result for T21, whereas this percentage was equal to $0.58 \%$ in 2013 .

Cf-DNA test after NT screening has changed women's decision for adding biochemistry test as a part of traditional screening tool. The percentage of women with combined test among women with NT screening has remained around 30\% throughout the years. The main difference over the years has appeared among women older than 35 years. In the group of $35-37$ years old women, this percentage decreased from $82.86 \%$ in 2013 to $57.69 \%$ in 2016. Among women of age 37 years or more with NT screening, $48.00 \%$ had also biochemistry test in 2013 and $23.94 \%$ decided for it in 2016 .

The average age of women with cf-DNA testing has been similar in the last 4 years (2013: 36.7 years, 2014: 36.5 years, 2015: 36.6 years and 2016: 36.2 years). In the period 2013 - 2016, half of our cf-DNA tests were performed in 2016. Out of total 157 women with cf-DNA tests in 2016, 84 (35.50\%) were of advanced maternal age ( 37 years or more), 36 (22.93\%) were HR for T21 based on prior screening, 59 (37.58\%)were HR regarding age but low risk according to prior screening, $62(39.49 \%)$ had low risk regarding age and prior screening.

The number of invasive procedures in Slovenia is still high despite well-established NT screening performed from year 1998 on. The percentage of women with invasive procedures among the ones with NT screening was $5.71 \%$ in $2013,5.30 \%$ in $2014,4.99 \%$ in 2015 , and $4.52 \%$ in 2016. However, this percentage decreased over the years in two age groups: for women of age $35-37$ years it was $9.66 \%$ in 2013 and $5.54 \%$ in 2016 , for women aged 37 years or more it was $37.24 \%$ in 2013 compared to $26.74 \%$ in 2016.

In general, cf-DNA testing for all aneuploidies yielded 100\% sensitivity (95\% CI: $63.06 \%-100 \%$ ) and $99.66 \%$ specificity (95\% CI: $98.14 \%-99.99 \%)$ with the positive predictive value of $88.89 \%$ (95\% CI: $51.75 \%-99.72 \%$ ). Sensitivity and specificity for only T21 are $100 \%$.

Conclusions. Our results confirmed that prenatal cf-DNA testing represents highly accurate approach in advanced screening of most common aneuploidies. The number and percentage of tests has been increasing. The average age of women with cf-DNA testing remains similar throughout the years. The expected decline in number of invasive procedures among HR pregnant women due to their age and/or prior NT screening results is to be continued. However, the ultrasound evaluation of the foetus determines which further test or procedure will follow.

\section{$\mathrm{L}-4$}

\section{Cff-DNA based NIPTs in Hungary}

\section{Olga Torok}

Institute of Obstetrics and Gynecology, Faculty of Medicine, University of Debrecen, Debrecen, Hungary

Objective. Noninvasive prenatal testing (NIPT) via cellfree fetal DNA has been proved to be the most sensitive and specific screening option for fetal chromosomal aneuploidies. The cffDNA based NIPTs started to be implemented in Hungary in 2012 and by now at least seven tests have become available on the market. The objective of the study is to examine the impact of NIPTs on the uptake of invasive testing and on the detection rate of trisomy-21.

Methods. Information regarding the number of invasive testing was collected from the database of the National Health Care Insurance for the interval between 2012-2014 and directly from the different prenatal diagnostic centers for 2015-2017. The NIPT data were supplied by the national providers of five big international commercial laboratories covering more than 90 percent of the Hungarian market. The source of the detection rate (DR) for trisomy 21 was the Hungarian Congenital Abnormalities Registry (HCAR). 
Results. The uptake of different NIPTs has increased continuously in Hungary, 2884 tests were performed in 2015, 4058 in 2016 and 6757 in 2017. The number of invasive procedures carried out for cytogenetic testing showed a significant decrease from 6662 in 2012 to 4010 in 2017 , but this decrease is much smaller than could be expected from the utilization data of NIPTs. According to the HCAR database, the detection rate of trisomy 21 did not show any improvement if data from 2012 and 2016 are compared, $68 \%$ and $62 \%$ respectively. It has to be mentioned that the system of reporting to HCR underwent significant changes within this interval and it can also be assumed that the prenatally diagnosed cases were underreported in 2015 and 2016. The analysis of the data from 2017 is still ongoing.

Conclusion. Results reflect a lack of well-organized nationwide first tier screening program. A well-defined national strategy could only improve the selection of patients to whom NIPTs could be recommended and that would lead to a much more significant decrease in the number of invasive procedures.

$\mathrm{L}-5$

\section{First trimester screening of circulating C19MC microRNAs and the evaluation of their potential to predict the onset of preeclampsia and FGR}

\author{
Ilona Hromadnikova ${ }^{\mathrm{a}, \mathrm{b}}$, Katerina Kotlabova ${ }^{\mathrm{a}, \mathrm{b}}$, \\ Katarina Ivankova ${ }^{b}$, Ladislav Krofta ${ }^{b}$
}

\section{${ }^{a}$ Department of Molecular Biology and Cell Pathology, Third Faculty of Medicine, Charles University, Ruska 87, 10000 Prague, Czech Republic \\ 'Institute for the Care of the Mother and Child, Third Faculty of Medicine, Charles University, Podolske nabrezi 157/36, 14700 Prague, Czech Republic}

Objectives. A nested case control study of a longitudinal cohort comparing pregnant women enrolled at 10 to 13 gestational weeks was carried out to evaluate risk assessment for preeclampsia and FGR based on circulating placental specific C19MC microRNAs in early pregnancy.

Methods. The expression of placental specific C19MC microRNAs (miR-516b-5p, miR-517-5p, miR-518b, miR520a-5p, miR-520h, and miR-525-5p) was determined in plasma samples from pregnancies that subsequently developed preeclampsia $(n=21)$, FGR $(n=18)$, and 58 normal pregnancies using real-time PCR and comparative $\mathrm{Ct}$ method relative to synthetic Caenorhabditis elegans microRNA (cel-miR-39).

Results. Circulating C19MC microRNAs were up-regulated (miR-517-5p, $P=0.005$; miR-518b, $P=0.013$; miR520h, $P=0.021$ ) or showed a trend toward up-regulation in patients destined to develop preeclampsia (miR-520a-5p, $P=0.067$; miR-525-5p, $P=0.073$ ). MiR-517-5p had the best predictive performance for preeclampsia with a sensitivity of $42.9 \%$, a specificity of $86.2 \%$, a PPV of $52.9 \%$ and a NPV of $80.6 \%$. The combination of all examined circulating C19MC microRNAs had no advantage over using only the miR-517-5p biomarker to predict the occurrence of preeclampsia (a sensitivity of $20.6 \%$, a specificity of $90.8 \%$, a PPV of $44.8 \%$, and a NPV of $76.0 \%$ ).

Conclusions. Up-regulation of miR-517-5p, miR-518b and miR-520h was associated with a risk of later development of preeclampsia. First trimester screening of extracellular miR-517-5p identified a proportion of women with subsequent preeclampsia. No circulating C19MC microRNA biomarkers were identified that could predict later occurrence of FGR.

Acknowledgement: Supported by the Charles University research programmes Prvouk P32 and Progres Q34.

L-6

\section{Follow-up of gestational trophoblastic disease/ neoplasia via quantification of circulating nucleic acids of placental origin using C19MC microRNAs, hypermethylated RASSF1A and SRY sequences}

\author{
Ilona Hromadnikova ${ }^{\mathrm{a}, \mathrm{b}}$, Katerina Kotlabova ${ }^{\mathrm{a}, \mathrm{b}}$, \\ Ladislav Kroftab ${ }^{b}$ Filip Hron ${ }^{b}$
}

${ }^{a}$ Department of Molecular Biology and Cell Pathology, Third Faculty of Medicine, Charles University, Ruska 87, 10000 Prague, Czech Republic

${ }^{b}$ Institute for the Care of the Mother and Child, Third Faculty of Medicine, Charles University, Podolske nabrezi 157/36, 14700 Prague, Czech Republic

Aims. To evaluate the effectiveness of placental specific markers, extracellular fetal DNA (SRY and hypermethylated RASSF-1A sequences) and circulating C19MC microRNAs (miR-516-5p, miR-517-5p, miR-518b, miR-520a-5p, miR-520h, miR-525 and miR-526a) for the diagnosis and consecutive follow-up of gestational trophoblastic disease/neoplasia.

Results. Increased levels of extracellular fetal DNA and C19MC microRNAs were detected in patients with active disease when compared with the period when the patients reached remission of the disease. The positive correlation between plasma levels of hypermethylated RASSF1A sequence, C19MC microRNAs and hCG serum levels was found. MiR-520a-5p had the best performance to detect patients with active disease (PPV of 100\% at a null FPR). MiR-516-5p and miR-525 were able to diagnose $100 \%$ of women with active disease at the FPR 3.9\%/7.7\%.

The overall predictive capacity of single miR-526a (81.8\% at null FPR), miR-517-5p (90.9\% at $15.4 \%$ FPR), miR-518b (100\% at 38.5\% FPR) and miR-520h (90.9\% at $26.9 \%$ FPR) biomarkers to detect active disease cases was slightly lower. Transient increase of C19MC microRNA 
plasma levels after the first cycle of chemotherapy indicated the decay of placental trophoblast residual tissue.

Conclusion. The increased levels of extracellular fetal DNA and placental specific C19MC microRNAs are associated with gestational trophoblastic disease/neoplasia.

Screening of extracellular placental specific biomarkers may represent an additional option to identify a significant proportion of women with active disease and to monitor the therapy response. Non-invasive follow-up of the decomposing residual tissue in the form of extracellular nucleic acids of placental origin packed into apoptotic bodies derived from placental trophoblasts is available.

Acknowledgement: Supported by the Charles University research programmes Prvouk P32 and Progres Q34.

\section{$\mathrm{L}-7$}

\section{Electrical storm in the brain and in the heart: Epilepsy and Brugada syndrome}

\section{Zoltan Csanadi}

Institute of Cardiology, Faculty of Medicine, University of Debrecen, Debrecen, Hungary

This is a report on the coincidence of two ion channel disorders with autosomal dominant inheritance: the Brugada syndrome, a potentially fatal cardiac condition, and cryptogenic focal epilepsy likely due to a neurological channelopathy. Clinical features of the patient shared the risk factor characteristics of the sudden unexplained death in epilepsy syndrome (SUDEP). This case set the stage for speculation on the potential interaction between ion channel abnormalities in the heart and in the brain. Further, implications for cardiac evaluation of patients at an increased risk for SUDEP is discussed.

$L-8$

\section{Non-invasive prenatal biomarkers for fetal heart defects - circulating nucleic acid and protein biomarkers in maternal blood}

$$
\text { Orsolya Biro a, Balint Nagy }
$$

\section{aFirst Department of Obstetrics and Gynaecology, Semmelweis University, Budapest, Hungary \\ ${ }^{b}$ Department of Human Genetics, Faculty of Medicine, University of Debrecen, Debrecen, Hungary \\ Correspondence to: biro.orsolya@noi1.sote.hu}

Background. Congenital heart defects (CHDs) are the most common fetal malformations. Prenatal ultrasonography is routinely applied for the screening of CHD but many factors influence its diagnostic accuracy. The introduction of new biomarkers could facilitate the identification of high-risk pregnancies.
Objective. We reviewed expression studies of maternal blood-derived cell-free nucleic acids and proteins in pregnancies affected by CHD. NIPT techniques which enabled the detection of heart-related chromosomal aberrations were also discussed.

Methods. Scientific literature databases were screened for studies where the levels of potential CHD biomarkers were measured in maternal blood samples. Available NIPT tests were collected from the providers' resources.

Results. To date, there are nine CHD-associated chromosomal abnormalities (five aneuploidies, and four microdeletions) which are included in different NIPT panels. We found eight studies from which five included the analysis of specific cell-free RNA expression profile and three measurements of protein levels.

Conclusion. Most of the common heart-related chromosomal aberrations can be diagnosed by NIPT. Specific cell-free RNAs and circulating proteins seem to be potential biomarkers for fetal CHDs. The application of these new biomarkers could improve the detection rate at early pregnancy, making it possible to provide optimal perinatal and perioperative management.

L-9

\section{Maternal methylenetetrahydrofolate reductase (MTHFR) polymorphisms: Risk factors for congenital heart disease?}

\section{Orsolya Biro ${ }^{a}$, Janos Rigo Jr. ${ }^{a}$, Balint Nagy ${ }^{\mathrm{b}}$}

\author{
aFirst Department of Obstetrics and Gynaecology, Semmelweis \\ University, Budapest, Hungary \\ ${ }^{b}$ Department of Human Genetics, Faculty of Medicine, University \\ of Debrecen, Debrecen, Hungary \\ Correspondence to: biro.orsolya@noi1.sote.hu
}

Background. Maternal MTHFR polymorphisms have been associated with higher risk for miscarriage, preeclampsia, spina bifida and congenital heart disease (CHD). MTHFR is responsible for the conversion of folic acid to the biologically active 1-methylfolate (5-MTHF), thus reduced enzyme can have severe consequences. Our aims were to assess the frequency of the two most common MTHFR polymorphisms (C677T and A1298C) in our Hungarian study group and to evaluate the association between these alterations and the risk for CHD.

Methods. Blood samples were collected from 40 pregnant women, 20 whose fetus was diagnosed with CHD and 20 healthy controls. DNA was isolated from buffy coat and FRET-based real-time PCR were performed using MTHFR C677T and A1298C specific probes. Genotypes were determined by melting curve analysis and frequencies were determined. The odds ratio (OR) and $95 \%$ confidence interval were calculated for CHD in each case.

Results. The frequencies for each genotype in all samples were CT/aa: 22.5\%, TT/aa: $15 \%, \mathrm{CC} / \mathrm{ca}: 15 \%, \mathrm{CC} / \mathrm{cc}$ : 
$22.5 \%, \mathrm{CT} / \mathrm{ac}: 12.5 \%$, and CC/aa: $12.5 \%$. The frequencies for each genotype in CHD vs control group: CT/aa: $30 \%$ vs. $15 \%, \mathrm{TT} /$ aa: $10 \%$ vs. $20 \%, \mathrm{TT} / \mathrm{ca}: 15 \%$ vs. $15 \%, \mathrm{CC} / \mathrm{cc}$ : $25 \%$ vs. $20 \%$, CT/ac: $15 \%$ vs. $10 \%$, and CC/aa: $5 \%$ vs. $20 \%$. The combined OR of MTHFR heterozygous, compound heterozygous or homozygous vs. wild type genotype was 4.75 for CHD, but the finding was not statistically significant.

Conclusion. We showed that MHTFR polymorphisms are relatively common in Hungarian women: only $12.5 \%$ of the samples were not affected by any of the alterations. The "less frequent" A $1298 \mathrm{C}$ is occurred in $50 \%$ of the samples, at the same rate as C677T.We have not found significant associations between the investigated polymorphisms and CHD, which may be due to the protective effect of periconceptional maternal supplement of bioavailable 5-MTHF against fetal abnormalities.

Key words: congenital heart disease, maternal circulation, cell-free nucleic acids, biomarkers

$\mathrm{L}-10$

\section{Circulating microRNAs and tumors}

\section{Peter Igaz}

\section{$2^{\text {nd }}$ Department of Medicine, Semmelweis University, Budapest, Hungary}

MicroRNAs (miRNAs) were discovered as the endogenous mediators of RNA interference. Since their discovery, miRNAs were shown to be involved in the regulation of gene expression mostly at the posttranscriptional level via affecting several basic physiological and pathological processes. MiRNAs belong to the epigenetic machinery. Differential expression of tissue miRNAs were described in several diseases including tumors. Both solid and hematologic tumors have been shown to display significant alterations in miRNA expression. miRNAs were established as markers of malignancy and prognosis. Recent findings have shown that miRNAs are found in body fluids. The release of miRNAs can be via a passive route (necrosis, inflammation) or by active secretion either in membrane vesicles (exosomes, microvesicles) or in association with macromolecular complexes (Argonaute 2 protein, or high-density lipoprotein). The released miRNA can affect neighboring and immune cells and thereby might contribute to tumor progression, invasion and metastasis formation. The circulating miRNAs of the blood can be exploited as a form of minimally invasive liquid biopsy, where miRNAs deliver epigenetic gene expression information from various tissues. Several studies have shown that the differential expression of circulating miRNA might be exploited in the establishment of malignancy that can be mostly significant in tumors where the histological analysis of malignancy is difficult (e.g. follicular thyroid tumors and adrenal tumors). Our research group has been involved in several studies on tissue and circulating miRNA expression in adrenal tumors.
$\mathrm{L}-11$

\section{The role of circulating cell-free DNA as a cancer biomarker}

\author{
Gergo Pappa , Ilona Kovalszky ${ }^{\mathrm{a}}$, Csaba Bodor ${ }^{\mathrm{a}, \mathrm{b}}$
}

${ }^{a}{ }^{\text {st }}$ Department of Pathology and Experimental Cancer Research, Semmelweis University, Budapest, Hungary

${ }^{b}$ MTA-SE Lendület Molecular Oncohematology Research Group, Budapest, Hungary

Correspondence to: papp.gergo@med.semmelweis-univ.hu

Circulating cell-free DNA (cfDNA) refers to short fragments of acellular nucleic acids detectable in almost all body fluids, including blood, urine, saliva, pleural effusions or cerebrospinal fluid, and is involved in various physiological conditions and pathological phenomena such as coagulation, immunity, aging, diabetes, myocardial infarction and cancer. In cancer patients, a fraction of cfDNA arises from tumors into the blood, termed circulating tumor DNA (ctDNA), and may harbor the same mutations and genetic alterations as those of the primary tumor. The concentration of ctDNA increases, and structural, sequence, and epigenetic changes to DNA can be observed through the disease process and during therapy. Thus, ctDNA potentially provides an opportunity for non-invasive assessment of cancer. Current advances in ctDNA analysis methods will conceivably lead to the development of a liquid biopsy tool for the diagnosis, prognosis, and therapy response monitoring, besides determining the emergence of drug resistance, and quantifying minimal residual disease. The molecular profiles collected from ctDNA can be further complemented with those obtained through analysis of circulating tumor cells (CTCs), as well as RNA, proteins, and lipids contained within vesicles, such as exosomes. Here, we are focusing on the different types of circulating biomarkers under investigation, describing the latest advances in their development and application to clinical settings, as well as challenges that researchers face in the procedure. In this presentation, we are highlighting several recent, key studies in the ctDNA area and confer future advances which would further enhance care for cancer patients.

$\mathrm{L}-12$

\section{Free nucleic acids in ovarian cancer}

\section{Robert Poka}

\section{Institute of Obstetrics and Gynecology, Faculty of Medicine, University of Debrecen, Debrecen, Hungary}

Ovarian cancer has the highest mortality among female pelvic malignancies and its incidence is on the rise in most developed countries. Due to its natural history and anatomical exposure early spread is a typical feature and results in $70 \%$ of cases being diagnosed in advanced stages. Despite tremendous efforts in improving surgical, 
chemotherapeutic, biological and targeted therapeutic interventions that have been made over recent decades, most patients die of their disease. Screening for ovarian cancer and that for susceptibility to ovarian cancer is an unmet medical need. Standard treatment methods have achieved their plateau in curative efficacy and practically restricted to cases with complete surgical resection or microscopic residual disease and sensitivity to platinumbased chemotherapy. Screening, early diagnosis, optimal timing of primary/secondary surgery and chemotherapy of platinum-resistant disease, early diagnosis of recurrence as well as the case for additional treatment modalities (i.e. biological and targeted therapies) are in the focus of development in the management of ovarian cancer. All these aspects of ovarian cancer are to be reviewed in this presentation with comprehensive literature overview of free nucleic acid investigations.

\section{L-13}

\section{Evaluation of circulating exosomal microRNAs in benign and malignant adrenocortical tumors}

\author{
Pal Perge ${ }^{\mathrm{a}}$, Henriett Butz ${ }^{\mathrm{b}}$, Raffaele Pezzanic, Irina Bancos ${ }^{\mathrm{d}}$, \\ Zoltan Nagy ${ }^{\mathrm{a}}$, Abel Decmann ${ }^{\mathrm{a}}$, Michaela Luconie, \\ Massimo Mannellie, Edit I. Buzas ${ }^{\mathrm{f}}$, Miklos Toth ${ }^{\mathrm{a}}$, \\ Marco Boscaroc, Attila Patocs ${ }^{\mathrm{b}, \mathrm{g}}$, Peter Igaz ${ }^{\mathrm{a}, \mathrm{f}}$
}

\begin{abstract}
${ }^{a} 2^{\text {nd }}$ Department of Medicine, Semmelweis University, 1088 Budapest, Szentkiralyi str. 46, Hungary
\end{abstract}

${ }^{b}$ Molecular Medicine Research Group, Hungarian Academy of Sciences and Semmelweis University, 1088 Budapest, Szentkiralyi str. 46, Hungary

'Endocrinology Unit, Department of Medicine, University of Padua, Via Ospedale, 105, 35128 Padova, Italy

${ }^{d}$ Division of Endocrinology, Diabetes, Metabolism and Nutrition, Department of Internal Medicine, Mayo Clinic, 200 First Street SW, Rochester, MN 55905 USA

'Department of Experimental and Clinical Biomedical Sciences, Endocrinology Unit, University of Florence, VialePieraccini 6, 50139 Florence, Italy

${ }^{f}$ Department of Genetics, Cell- and Immunobiology, Semmelweis University, 1089 Budapest, Nagyvarad ter4., Hungary

g"Lendulet-2013" Research Group, Hungarian Academy of Sciences and Semmelweis University, 1088 Budapest, Szentkiralyi str. 46, Hungary

Introduction. MicroRNAs (miRNA) have been described in patients with adrenocortical tumors, however, there is no available plasma biomarker for the preoperative diagnosis of adrenocortical malignancy. Furthermore, the expression of miRNAs in relation to the cortisol-secreting activity of adrenocortical tumors has not yet been investigated.

Aims. The first aim of this study was to investigate the expression of exosomal miRNAs and their potential diagnostic utility in adrenocortical tumors. The second objective was to examine the expression of plasma exosomal miRNAs in patients suffering from non-functioning adrenocortical adenoma (NFA), cortisol-producing adrenocortical adenoma (CPA) and cortisol-producing adrenocortical carcinoma (CP-ACC).

Method. Exosomes were isolated either by performing ultracentrifugation or by applying Total Exosome Isolation Kit. Preoperative plasma exosomal samples of 6 adrenocortical adenomas (ACA) and 6 adrenocortical carcinomas (ACC) were first screened by TaqMan Human MicroarrayA-cards two miRNAs (miR-101 and miR-483-5p) were subjected to validation in 18 ACAs and 16 ACCs by targeted RT-qPCR. Based on previous results, the expression of miR-22-3p, miR-27a-3p, miR-210$3 \mathrm{p}$, miR-320b and miR-375 were investigated in 13 NFA, $13 \mathrm{CPA}$ and $9 \mathrm{CP}-\mathrm{ACC}$ samples. Exosome preparations were also investigated by transmission electronmicroscopy, flow cytometry and dynamic light scattering.

Results. We detected significant overexpression of miR-101 and miR-483-5p in ACC compared to ACA in the validation cohort. Receiver operator characteristics of data revealed miR-483-5p to have the highest diagnostic accuracy (area under curve 0.965 ), the sensitivity and the specificity were 87.5 and 94.44 , respectively. We identified significant overexpression of 3 miRNAs in both CPA and CP-ACC versus to NFA: miR-22-3p, miR-27a-3p and miR-320b. miR-320b has been significantly overrepresented in CP-ACC compared to CPA. miR-210-3p was significantly overrepresented only in CP-ACC relative to NFA. Significant correlation was observed between circulating miRNA expression and urinary free cortisol concentrations for miR-22-3p, miR-27a-3p and miR-320b and cortisol level after low dose dexamethasone test for miR-22-3p and miR-320b. Furthermore, miR-27a-3p was significantly triggered by low dose dexamethasone test.

Conclusion. Exosomal miR-101 and miR-483-5p might be potential preoperative biomarkers of ACC. Furthermore, exosomal miRNAs are dissimilarly expressed in hormonally inactive and cortisol-secreting adrenocortical neoplasm.

$\mathrm{L}-14$

\section{Analysis of oncogenic mutations from liquid biopsy samples: Potentials and limitations in oncology}

\section{Gabor Mehes, Judit Bedekovics, Attila Mokanszki}

\author{
Department of Pathology, Faculty of Medicine, University of \\ Debrecen, Debrecen, Hungary \\ Correspondence to: gabor.mehes@med.unideb.hu
}

Mutation profiling for clinically relevant cancer related genes gained crucial significance in therapeutic decisions in oncology. Further to the diagnostic or prognostic classification an increasing need for therapy associated gene 
targets evolved. The most frequent epithelial cancers (lung adenocarcinoma, colorectal carcinoma, etc.) are now routinely investigated for EGFR, RAS and BRAF sequences in order to determine sensitivity for EGFR-targeted therapeutic approaches. DNA extraction and new generation sequencing (NGS) is well established from formaldehyde fixed and paraffin embedded (FFPE) tissues following histopathological examination. The availability of new kinase inhibitors requires the analysis of increasing numbers of cell signaling gene exons predicting failure of response.

For these reasons, an increasing need for non-invasive follow-up and even more for the early demonstration of newly aquired mutations during the course of the disease evolved. Peripheral blood derived circulating free DNA (cfDNA) analysis is a highly promising option as retrieval of biopsy samples from the recurrent/metastatic tumor is limited. Cancer genetic profiling from the serum - also termed as liquid biopsy - is enabled by refined DNA isolation and high sensitivity-high throughput sequencing technologies. The laboratory method of choice, the sampling modalitiy and timing all critically determine the quality of the test result. Nevertheless, biological/pathological aspects, such as tumor size, localization, necrosis, inflammatory/reparatory changes and intratumor heterogeneity eminently influence the composition of the cfDNA provided for analysis at a certain date.

\section{$\mathrm{L}-15$}

\section{Our first experiences with testing EGFR T790M resistance mutation in liquid biopsy}

\author{
Ilona Kovalszky, Laszlone Olah, Krisztina Csaszar, \\ Krisztina Egedi, Csaba Bodor \\ $1^{\text {st }}$ Department of Pathology \& Experimental Cancer Research, \\ Semmelweis University Budapest, Hungary \\ Correspondence to: koval@korb1.sote.hu
}

Backgroung. EGFR tyrosine kinase receptor inhibitors represent a cornerstone in the therapy of advanced nonsmall cell lung cancers. These small molecules interfere with the activation of the receptor by interacting with the ATP binding site of their kinase domain. In 2015, FDA approved gefitinib in $1^{\text {st }}$ line therapy in unresectable advanced tumors. Progression free survival with the treatment of gefitinib proved to be significantly longer with much less side effects, than that of chemotherapy.

However, after a while the effect of TK inhibitors declines as a result of a new resistance mutation, occurring on exon 20 of the receptor. A $3^{\text {rd }}$ generation TK inhibitor effective against the T790M mutation reestablishes the inhibition of EGFR, but the presence of resistance mutation has to be proved for approval of the therapy. As in most of the cases removal of new tumor specimen is cumbersome, analysis of cell free circulating DNA offers an alternative approach for T790M mutation detection.

To this end, two years ago our laboratory decided to introduce the detection of the EGFR T790M mutation from liquid biopsy specimens.

Methods. To save the integrity of cfDNA we started to collect blood specimens in Streck tubes. cfDNA is isolated by Mad Max Cell-free DNA isolation Kit (Thermo Fisher)from $4 \mathrm{ml}$ of plasma.T790M mutations are detected using the Therascreen EGFR Pyro Kit (Qiagen) and Pyromark sequencer.

Results. In 2017, 8.8\% (57 cases) of all lung adenocarcinomas, studied, carried an EGFR activating mutation and clinicians requested detection of T790M from 38 cases. Out of these, 8 specimens proved positive for the T790M mutation.

In the first three month of 2018, 11 liquid biopsies were analyzed and 6 of them carried a T790M mutation indicating the improvement in sensitivity of our technique.

Conclusions. Comparison of the sensitivity of pyrosequencing and the recently introduced digital droplet PCR (BioRad) method proved excellent agreement above $1 \%$ variant allele frequency (VAF) of T790M, however droplet PCR was also capable to detect T790M mutations with VAFs lower than $1 \%$, indicating the superiority of this method.

\section{$\mathrm{L}-16$}

\section{Cell-free tumor load as possible predictive marker for TKI response in NSCLC therapy}

\author{
Vilmos Adleffa, Jillian Phallen ${ }^{\mathrm{a}}$, Alessandro Leal ${ }^{\mathrm{a}}$, \\ Brian Woodward ${ }^{b}$, Patrick M. Forde ${ }^{a}$, Kristen A. Marrone ${ }^{a}$, \\ Julie R. Brahmer ${ }^{a}$, Jacob Fiksel ${ }^{a}$, Stephen Cristiano ${ }^{a}$, \\ Daniel C. Bruhm ${ }^{\mathrm{a}}$, Elizabeth Weihe ${ }^{\mathrm{b}}$, Valsamo Anagnostou ${ }^{\mathrm{a}}$, \\ Robert B. Scharpfa, Hatim Husain ${ }^{b}$, Victor E. Velculescu ${ }^{\mathrm{a}}$
}

\author{
aThe Sidney Kimmel Comprehensive Cancer Center, Johns \\ Hopkins University School of Medicine, Baltimore, MD 21287, \\ USA \\ ${ }^{b}$ Division of Hematology and Oncology, Moores Cancer Center, \\ University of California, San Diego, La Jolla, CA 92093, USA \\ Correspondence to: vadleff1@jhmi.edu
}

Widespread applicability of genomic profiling of ctDNA as biomarker will be strongly influenced by the development of innovative and cost-effective analytical methods. We previously described an ultrasensitive targeted error correction sequencing method (TEC-Seq) for cancer detection in cfDNA. Using high coverage targeted sequencing $(30,000 x, 58$ gene panel), and optimized sequencing and bioinformatics, TEC-Seq was able to achieve a $\sim 60-70 \%$ TPR on a cohort of 200 early stage cancer patients. As a proof of principle here we applyTEC-Seqfor monitoring disease progression during first-line TKI therapy.

16 patients with metastatic NSCLC undergoing targeted therapy had serial blood draws obtained immediately prior to $\left(\mathrm{t}_{0}\right), 1-3$ weeks after the treatment $\left(\mathrm{t}_{1}\right)$, and 
at a number of timepoints until disease progression. We defined cell-free tumor load (cfTL) as the most abundant targeted clone a teach timepoint. The two tiered direct detection of sequence and copy number alterations from TEC-Seq and from WG sequence analyses of chromosomal changes identified at least one alteration for each patientat $\mathrm{t}_{0}$. The longitudinal analyses revealed that patients with a radiographic response to therapy had a significant drop intheir cfTLvalue, as well as in the number of detectable mutations and aneuploidy scores overall. As early as the first post-treatment liquidbiopsy time points $\left(\mathrm{t}_{1}\right)$, taken on average 38 days earlier than the corresponding imaging assessment, the cfTL values indicated a drop in the mutant allele fraction from an average of $3.59 \%$ to $0.13 \%$, $(P<0.05)$, while the radiographicnon-responders had limited to no changes in their cell-free tumor load. Stratified by the cfTL score changes at $t_{1}$, molecular responders had a significantly longer progression-free survival than the nonresponders ( 12.4 vs 1.7 months, $P<0.001)$ on this small cohort of NSCLC patients, suggesting a possible predictive marker value of the cfTL for the TKI therapy. The inceptive ctDNA dynamics at 1-3 week spost-treatment can be thus used as a very early predictive biomarker, and it is a spredictive in this setting as the first CT imaging.

\section{$\mathrm{L}-17$}

\section{Non-invasive detection of biomarkers in plasma of cancer patients using droplet digital PCR - overview, first experiences and perspectives}

\author{
Zora Lasabovaa, Tatiana Burjanivovab, \\ Bibiana Malicherova a, , Marian Grendard, Barbora Vanovaa,b, \\ Lukas Plank ${ }^{\mathrm{a}, \mathrm{c}}$
}

\author{
${ }^{a}$ Division Oncology, Biomedical Center Martin, Comenius \\ University in Bratislava, Jessenius Faculty of Medicine in Martin, \\ Slovak Republic \\ ${ }^{b}$ Department of Molecular Biology, Comenius University in \\ Bratislava, Jessenius Faculty of Medicine in Martin, Slovak \\ Republic \\ 'Department of Pathological Anatomy, Comenius University in \\ Bratislava, Jessenius Faculty of Medicine and University Hospital \\ in Martin, Slovak Republic \\ ${ }^{d}$ Department of Bioinformatics, Biomedical Center Martin, \\ Comenius University in Bratislava, Jessenius Faculty of Medicine \\ in Martin, Slovak Republic \\ Correspondence to: lasabova@jfmed.uniba.sk
}

The Cancer Genome Atlas (TCGA) project revealed an immeasurable wealth of data concerning the genetic and epigenetic driver and passenger changes within cancer genomes. Even though the basic multistep clonal model of carcinogenesis was not challenged, the results from TCGA have shown that the number of mutated genes vary between different types of tumors as well as from patient to patient with the same tumor type. Moreover, the carcinogenesis as a multistep process involves in addition to accumulation of driver mutations also epigenetic changes, somatic variations in DNA copy number, interactions with tumor microenvironment in general and immune system response in particular as well. Advances in high-throughput sequencing methods and non-invasive approaches focus the recent research on the finding of new biomarkers for patient's follow-up and early detection of relapse and on development of new screening methods for early non-invasive cancer detection.

Recently, the most successfully applied methods are approaches that concentrate on the follow-up by non-invasive detection of actionable mutations. Droplet digital PCR is very sensitive method that can be used for noninvasive analysis of tumor genotype and for identification of actionable mutations to monitor therapeutic efficacy in cancer patients. This approaches enable targeted mutational analysis of selected genes for presence or absence of specific mutations and are suitable for patients whose harbor these specific mutations. However, for patients whose harbor less frequent mutations or the mutation burden is under the limit of detection used by standard NGS approaches in tissues, more universal markers are necessary to be identified in order to detect relapse as early as possible. These markers can rely on general properties of cancer cells such as hypermethylation of specific genes involved in early carcinogenesis, somatic copy number variations ( $\mathrm{sCNV}$ ) or global hypomethylation.

We have first experience with droplet digital PCR in non-invasive detection of $B R A F \mathrm{~V} 600 \mathrm{E}$ mutations in plasma of patients with malignant melanoma. Detection of specific methylated sequences could be universal markers to follow early relapse, especially in patients without identified driver mutations. Our next focus is development of non-invasive detection of methylation in colorectal cancer patients after disease recurrence using standard real-time PCR compared with droplet digital PCR. These methods are in development. The combined approaches using different markers can be a useful tool for early cancer detection and disease recurrence in addition to conventional screening approaches.

Acknowledgement: This study was supported by the Biomedical Center Martin (IMTS 26220220187), which is co-financed from EU sources, and by the projects of the Slovak Research and Development Agency no. APVV- 140273 and VEGA 1/0380/16. 
$\mathrm{L}-18$

\section{Correlation of plasma mitochondrial DNA copy number and status of ovarian cancer patients}

\author{
Judit Keseru ${ }^{\mathrm{a}}$, Beata Soltesz ${ }^{\mathrm{a}}$, Janos Lukacs ${ }^{\mathrm{b}}$, Robert Poka ${ }^{\mathrm{b}}$, \\ Balint Nagy ${ }^{a}$
}

${ }^{a}$ Department of Human Genetics, Faculty of Medicine, University of Debrecen, Debrecen, Hungary

'IInstitute of Obstetrics and Gynaecology, Faculty of Medicine, University of Debrecen, Debrecen, Hungary

Introduction. Mitochondria are important organelles in ATP synthesis of cells in aerobic conditions, and are involved in various different cell processes like cell proliferation, and apoptosis. Their copy number variations were connected to many different pathological conditions like development of many types of cancer, e.g. ovarian cancer. That is why we measured the mtDNA copy numbers in the plasma in ovarian cancer patients and healthy controls.

Materials and Methods. We involved 53 healthy controls (avarage age $57.45 \pm 10.61$ years) and 24 ovarian cancer patients (average age $61.33 \pm 12.72$ years) in the study. Blood was drawn into EDTA tubes and plasma was separated by centrifugations. MtDNA copy numbers were determined by Human Mitochondrial DNA (mtDNA) Monitoring Primer Set kit (Takara, Japan) with real-time PCR.

Results. The mtDNA copy number was $43.55 \pm 13.69$ in the healthy control group. We divided the ovarian cancer patients into groups according to their FIGO classification and their cancer stage. The copy numbers were the followings: FIGO I. (early-stage ovarian cancer) $32 \pm 8.27$; FIGO III. and IV (advanced-stage ovarian cancer) $37.19 \pm 13.75$ (FIGO III. 33 \pm 5.66 ; FIGO IV. $42 \pm 18.87$ ). Student's unpaired t-test was performed. No significant difference was found among the early-stage and advanced-stage patients or control group and advanced stage patients ( $P=0.3740$ and $P=0.1084$, respectively) but significant difference was found among control group and early-stage patients $(P=0.0290)$ in the plasma cellfree mtDNA copy numbers.

Conclusion. We determined the cell-free mtDNA copy numbers in the plasma of ovarian cancer patients and healthy controls and we found significant difference among control group and early-stage patients. We plan to extend the study to more ovarian cancer patients and also we plan exosome encapsulated mtDNA measurements.
L-19

\section{miR200a, miR200b and miR200c are promising candidate biomarkers in epithelial ovarian cancer}

\author{
Melinda Szilagyi-Bonizs ${ }^{\mathrm{a}}$, Eva Marton ${ }^{\mathrm{a}}$, Janos Lukacs ${ }^{\mathrm{b}}$, \\ Beata Soltesz ${ }^{\mathrm{a}}$, Eszter Janka ${ }^{\mathrm{c}}$, Andras Penyige ${ }^{\mathrm{a}}$, Robert \\ Poka ${ }^{b}$, Balint Nagy ${ }^{\mathrm{a}}$
}

\begin{abstract}
${ }^{a}$ Department of Human Genetics, Faculty of Medicine, University of Debrecen, Debrecen, Hungary

${ }^{b}$ Department of Obstetrics and Gynecology, Faculty of Medicine, University of Debrecen, Debrecen, Hungary

'Department of Dermatology, Faculty of Medicine, University of

Debrecen, Debrecen, Hungary
\end{abstract}

Ovarian cancer is the fifth most common form of cancer death among women. Developing a fast, reliable blood test would facilitate the early detection of ovarian cancer, which would also contribute to the improvement of survival chances. Screening circulating miRNAs proved to be reliable biomarkers in various cancers. MiRNAs are also dysregulated in ovarian cancer, however, only a few publications focus on their diagnostic role.

We screened 6 miRNAs in the plasma samples of healthy $(n=30)$, previously untreated epithelial ovarian carcinoma patients $(n=21$, FIGO stages III or IV) and patients with benign masses $(n=14)$. The relative amount of miRNAs was detected by qRT-PCR.

The expression levels of miR200a, miR200b and $m i R 200 c$ were elevated in the malignant samples compared to the healthy donors $(P<0.001)$. The level of $m i-$ $R 200 a$ was also higher in malignant than benign samples $(P<0.01)$. However, no significant difference was detected in the expression of miR205, miR483 and let $7 f$. ROC-AUC was the highest in the case of $m i R 200 a(0.875$, $95 \% \mathrm{CI}=0.76-0.99)$ and it was still promising in the case of $m i R 200 b(0.85,95 \% \mathrm{CI}=0.72-0.98)$ and $m i R 200 c(0.83$, $95 \% \mathrm{CI}=0.70-0.97)$. Spearman's rank correlation was relatively high between the expression values of $m i R 200 \mathrm{~b}$ and $\operatorname{miR} 200 c\left(\mathrm{r}_{\mathrm{s}}=0.858 ; P<0.001\right)$ and these miRNAs shared several target genes involved in cancer development according to target prediction analysis. The agreement of diagnostic tests based on miR200s and CA125 or HE4 proved to be weak according to Cohen's kappa values.

We conclude that $m i R 200 a$, miR200b and miR200c might be promising complementary markers of CA125 and HE4 in ovarian cancer. Moreover miR200a is also a reliable biomarker in the differential diagnosis of benign tumors. 
$\mathrm{L}-20$

\section{Cell-free, long non-coding RNA in the diagnosis of ovarian cancer}

\section{Beata Soltesz ${ }^{\mathrm{a}}$, Janos Lukacs ${ }^{\mathrm{b}}$ Edina Szilagyi ${ }^{\mathrm{a}}$, Robert Poka ${ }^{\mathrm{b}}$, Balint Nagy ${ }^{\mathrm{a}}$}

${ }^{a}$ Department of Human Genetics, Faculty of Medicine, University of Debrecen, Debrecen, Hungary,

'Institute of Obstetrics and Gynecology, Faculty of Medicine,

University of Debrecen, Debrecen, Hungary

Correspondence to: nagy.balint@med.unideb.hu

Background. Ovarian cancer is the one of leading causes of cancer-associated mortality among women. An optimalised and established non-invasive diagnostic method may contribute to the early diagnosis of this malignancy. The significant role of the long non-coding RNAs (lncRNAs) was described in the oncogenesis, metastasis and chemoresistance. Upregulation of the one of IncRNA, namely, Hox transcript antisense intergenic RNA (HOTAIR) was determined in the development of ovarian cancer cells.

Materials and Methods. Twenty previously untreated ovarian cancer patients $(60.60 \pm 10.84 \mathrm{y}$; FIGO stages III or IV) and 20 healthy controls (56.70 $\pm 14.51 \mathrm{y})$ were studied. EDTA-anticoagulated blood was drawn; RNA was isolated; cDNA was synthesised and the HOTAIR lncRNA expression were determined by using ExiLERATE LNA ${ }^{\mathrm{TM}}$ qPCR,for mRNA and long non-coding RNA (Exiqon, USA). Beta-actin was used as reference gene. Student ttest was used for the statistical calculations.

Results. Higher expression of HOTAIR was detected in the cell-free plasma samples of patients with ovarian cancer $(1.89 \pm 2.39)$ compared to healthy controls $(1.39 \pm 1.48)$, while the statistical analysis did not show significant difference.

Conclusion. This is the first HOTAIR expression determination from the cell-free plasma of ovarian cancer patients and healthy controls, according to our knowledge. No significant difference was determined in the expression of the analysed lncRNA between Hungarian ovarian cancer patient and healthy control groups.

\section{$\mathrm{L}-21$}

\section{NGS based ctDNA-liquide biopsy analysis in Turkish cancer patients}

\author{
Naci Cine ${ }^{\mathrm{a}}$, Nilufer Sertdemir ${ }^{\mathrm{a}}$ Duygu Yavuz ${ }^{\mathrm{a}}$, \\ Merve Gokbayraka, Eda Guzdolua , Gulhan Demira, \\ Devrim Cabuk ${ }^{\mathrm{b}}$, Ulas Isik ${ }^{\mathrm{b}}$, Ercan Ozden ${ }^{\mathrm{b}}$, Kazim \\ Uygun $^{b}$,Gorkem Aksu', Hakan Savlia
}

a Department of Medical Genetic, Faculty of Medicine, Kocaeli University, Izmit, Turkey

${ }^{b}$ Department of Medical Oncology, Faculty of Medicine, Kocaeli University, Izmit, Turkey

'Department of Radiation Oncology, Faculty of Medicine, Kocaeli University, Izmit, Turkey

Correspondence to: nacicine@yahoo.com

Introduction. Circulating tumor DNA (ctDNA) is noninvasive cancer mutation detection targets, which is fast and becoming an important complement to tissuespesific genotyping for diagnosis and define the resistance at cancer progression.

Liquid biopsies are based on the analysis of ctDNA which has been scattered from tumors and carryingtheirpossible disease based mutations. In this study we represented that capasity and perfection of the cfDNA-liquide biopsy test in Non-small cell lung cancer (NSCLC) and Colon cancer mutation detection.

Materials and Methods. Plasma preparation: Blood samples were collected into EDTA tubes following manufacturer's instructions. Plasma from blood sample was obtained by centrifugation at $2000 \mathrm{x}$ g for $10 \mathrm{~min}$ at 4 ${ }^{\circ} \mathrm{C}$, followed by another spin at $16,000 \mathrm{x}$ g for $10 \mathrm{~min}$ at $4{ }^{\circ} \mathrm{C}$ to remove any residual blood cells. cfDNA/FFPE DNA isolation: cfDNA was isolated from 10-mL of plasma using MagMAX Cell-Free DNA Isolation Kit following alternative protocol in User Guide. RecoverAll TM Multi-Sample RNA/DNA Isolation Workflow was used to isolate DNA from FFPE samples following standard protocol.

Library preparation: cfDNA Lung Assay was used to generate libraries from both cfDNA and FFPE DNA samples following standard user guide. Sequencing: Ion 520TM/Ion 530TM, followed by sequencing on Ion S5 system using Ion $520 \mathrm{TM} /$ Ion 530 chips.

Data analysis: Data analysis was performed in Torrent Suite using variantCaller cfDNA plugin.

Results and Discussion. In this study, we demonstrated that importance of the Non-small cell lung cancer (NSCLC) and Colon cancer mutation detection by cfDNA based liquide biopsy test in Turkish cancer patient. All good results have taken by large volume of blood sampling (10 mL plasma) and high amount of DNA input.After inslico analysis showed that lower frequency variants may detected by NGS workflow. We observed that liquide biopsy analysis highly powerfull tool for detection of the major cancer mutation. 
$\mathrm{L}-22$

\section{Deacetylation of JAK1 and its association with IL-6-induced inflammatory response in cancer}

\author{
Ozkan Ozden, Illhami Gok
}

Department of Bioengineering Central Campus, Faculty of Engineering and Architecture, Kafkas University, 36100, Kars, Turkey

Correspondence to:dnzgoki@gmail.com

Interleukin 6 (IL-6) is an immune response protein which is secreted primarily by T-cells and macrophages to fight with infection during trauma, burns, and other tissue damages. It is a pro-inflammatory cytokine critical in immunoregulation. Aberrant IL-6 signaling has been implicated in various diseases including inflammationassociated cancer. Anti-IL-6 agents have been sought as therapeutics for many cancer types. SIRT2 (sirtuin2) is a cytoplasmic and nuclear member of sirtuin family and plays important roles in the regulation of stress response, and cancer. It plays dual roles as a tumor suppressor and oncogene in various tumors. SIRT2 gene removed mouse develops tumors in many organs, including the liver and breast tissues. SIRT2 has been shown to deacetylate numerous substrates and the mechanisms how SIRT2 decreases inflammation and inflammation associated disorders has been poorly understood up-to-date. The purpose of the study was to investigate the roles of SIRT2 on IL-6 induced inflammatory response in mouse embryonic fibroblasts (MEFs) with SIRT2 gene removed and HeLa cervical cancer cell lines that overexpress SIRT2 gene. SIRT2 physically interacts with JAK1, which is one of the downstream proteins of IL-6. Overexpression of SIRT2 in HeLa cervical cancer cells decreases IL-6 induced inflammatory response by decreasing the activity of JAK1. Our results indicate that SIRT2 interacts and decreases the activity of JAK1 kinase; and ultimately, it decreases the activity of IL-6 induced JAK/STAT3 signaling cascade, suggesting that SIRT2 have an immune response regulatory function through IL-6 in cancer cells.
L-23

\section{Cell-free miRNAs as potential biomarkers in the diagnosis of ovarian cancer}

\author{
Beata Soltesz $z^{\mathrm{a}}$, Orsolya Birob, Janos Lukacsc, \\ Andras Penyige ${ }^{\mathrm{a}}$, Robert Poka', Balint Nagy ${ }^{\mathrm{a}}$
}

\author{
${ }^{a}$ Department of Human Genetics, Faculty of Medicine, University \\ of Debrecen, Debrecen, Hungary \\ ${ }^{b}$ Department of Obstetrics and Gynecology, Semmelweis \\ University, Budapest, Hungary \\ IInstitute of Obstetrics and Gynecology, Faculty of Medicine, \\ University of Debrecen, Debrecen, Hungary \\ Correspondence to: nagy.balint@med.unideb.hu
}

Background. Ovarian cancer is the fifth leading cause of cancer mortality among women due to the poor early diagnostic tools for the detection of this type of aggressive tumour. Cell-free microRNAs appear to be potential candidate biomarker in this early diagnosis.

Materials and Methods. Eleven ovarian cancer patients and 11 healthy controls were involved into the study. EDTA-anticoagulated blood was drawn; the microRNA was isolated with miRNeasy Mini kit (Qiagen, Germany) and cDNA was synthesised. The miR-103; miR-125, miR193b and miR-200b expression were determined by miRCURY Universal RT microRNA PCR Starter kit (Exiqon, USA). The results were normalised to the miRNA-93 reference gene. Student t-test was used for the statistical calculations.

Results. Significant difference in the expression of miR-103 $(P=0.001)$, miR-193b $(P=0.0236)$, miR-125 $(P=0.029)$ and miR-200b $(P=0.0273)$ was detected among the cancer patients and controls.

Conclusion. The expression of four different cell-free microRNAs was determined from the plasma of patients with ovarian cancer and healthy controls. Significant difference was found in the expression of the analysed microRNAs. In the near future, our study will be extended on higher number of cases.

$\mathrm{L}-24$

\section{Editing the epigenome using a comprehensive CRISPR/Cas9-based molecular toolbox}

\author{
Aleksandar Vojta, Goran Josipovic, Vanja Tadic, \\ Vlatka Zoldos
}

Department of Biology, Faculty of Science,University of Zagreb, Zagreb, Croatia

Correspondence to: vojta@biol.pmf.hr

The advent of the CRISPR/Cas9 technology revolutionized the field of genome engineering by providing a flexible platform for targeting almost any genomic region. Soon, fusions of the targeting moiety Cas 9 with various 
effector domains enabled the construction of a novel class of molecular tools useful for gene regulation and epigenetic modifications. We focused on targeted $\mathrm{CpG}$ methylation and demethylation of gene promoters, which is a major epigenetic mechanism controlling gene expression. First, we constructed a modular molecular toolbox for introducing targeted epigenetic modifications, which enabled easy deployment and application to biological problems. After the challenging step of creating active fusions between Cas 9 and DNMT3A or TET1 catalytic domains for $\mathrm{CpG}$ methylation/demethylation and careful characterization of the new molecular tools, we approached biological problems in the field of epigenetic regulation of protein glycosylation with special emphasis on the role of IgG glycosylation in inflammatory diseases. We are using this platform to reveal the underlying epigenetics responsible for the inflammatory phenotype common to many diseases with the aim of discovering robust biomarkers and also to pave the way for personalized and precise epigenetic therapy in the future.
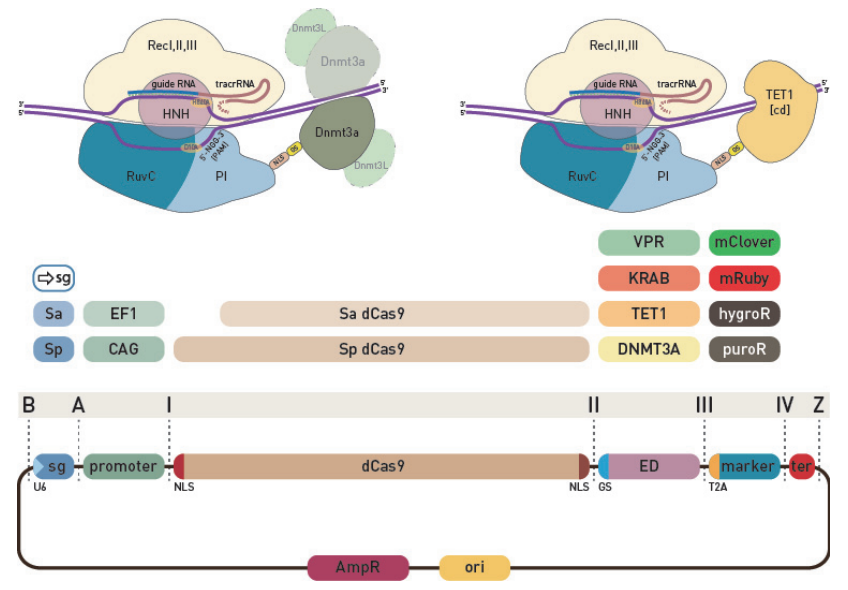

$\mathrm{L}-25$

\section{Are phages part of our immunity?}

\author{
Tomas Szemes ${ }^{\mathrm{a}, \mathrm{ce} e,}$, Andrej Balaz, ${ }^{\mathrm{b}, \mathrm{e},}$, Michal Kajsik ${ }^{\mathrm{a}, \mathrm{b}}$, \\ Iveta Gazdaricova ${ }^{b}$, Orsolya Biro ${ }^{d}$, Balint Nagy ${ }^{d}$, Jan Turna ${ }^{\mathrm{a}, \mathrm{b}}$, \\ Jaroslav Budis, ${ }^{\mathrm{b}, \mathrm{e}}$
}

\author{
${ }^{a}$ Faculty of Natural Sciences, Comenius University, Bratislava, \\ Slovak Republic \\ ${ }^{b}$ University Science Park, Comenius University, Bratislava, Slovak \\ Republic \\ 'Faculty of Mathematics, Physics and Informatics, Comenius \\ University, Bratislava, Slovak Republic \\ ${ }^{d}$ Department of Human Genetics, Faculty of Medicine, University \\ of Debrecen, Debrecen, Hungary \\ ${ }^{e}$ Geneton Ltd, Bratislava, Slovak Republic \\ Correspondence to: tomas.szemes@uniba.sk
}

Introduction. Several non-invasive prenatal tests (NIPT) use total DNA massively parallel sequencing. A portion of sequences are not mapped to human genome reference sequence and is filtered out in the NIPT analysis. Our aim was to determine source of unmapped reads. Preliminary data suggests presence of bacteriophage DNA in human circulation.

Materials and Methods. We carried out massively parallel sequencing of total DNA from plasma from group of healthy individuals and individuals undergoing antiviral treatment. DNA fragments which mapped to the human reference genome (version hg38) were filtered. We assigned each unmapped fragment a taxonomic label using metagenomic classifier Clark. Taxonomic composition of samples was compared and visualized using Krona graphs. We assembled filtered reads using assembler specialized for assembly of viral genomes called Savage.

Results. We identified presence of phage sequences in a small number of reads in studied samples of healthy individuals. In addition, we observed significant drop in phage sequences in individuals undergoing antiviral treatment. In the results we present breakdown of the identified phage data.

Conclusions. Preliminary data suggest presence of phage DNA in circulating free nucleic acids. We hypothesize that host immune system tolerates phages to assist in control and stabilization of microenvironment. More rigorous analysis needs to be carried out and supportive experiments are needed to confirm the finding as well as to study our hypotheses.

$\mathrm{L}-26$

\section{Sex differences in plasma deoxyribonuclease activity in rats}

\section{Emese Domonkos, Lubica Janovicova, Manuel Steinhardt, Tim Hopfner, Thomas Stehle, Peter Celec}

Institute of Molecular Biomedicine, Faculty of Medicine, Comenius University, Sasinkova 4, Bratislava, Slovak Republic

Correspondence to: domonkos.emese@gmail.com

Extracellular DNA is involved in the pathogenesis of several disorders including rheumatoid arthritis and systemic lupus. The prevalence of these autoimmune diseases is higher in women than in men, while the cause is unknown. Experiments proved that exogenous administration of deoxyribonuclease (DNase) might attenuate inflammation in lupus, sepsis or hepatorenal injury. Sex differences have been described in DNase activity in various tissues, but plasma DNase activity has not been compared between sexes yet. The aim of our study was to analyze plasma DNase activity in male and female rats. As in two independent experiments sex differences have been found we have also tested the role of testosterone and estradiol in gonadectomized male and female rats to prove whether the sex differences are caused by sex hormones.

Plasma samples from 18-month old aging rats of both sexes were analyzed. Male rats were either orchidectomized or sham-operated prior to puberty onset, and 
female rats were intact. In the next experiment, plasma samples from one-year old rats were used. Female rats were either ovariectomized or sham-operated in adulthood, while males were left intact. Half of the ovariectomized and sham-operated females were treated with estradiol or letrozole, an aromatase inhibitor, respectively, for 3 weeks. Others received vehicle. DNase activity in the plasma samples was measured using single radial enzyme diffusion assay.

Female rats displayed approximately 2-fold higher DNase I activity in comparison to male rats in both experiments. Neither orchidectomy in males, nor ovariectomy in females affected DNase I activity. Inhibition of estradiol production in sham-operated females resulted in tendency to decrease DNase I activity, but estradiol supplementation in ovariectomized rats had no effect.

Our results show sex differences in DNase I activity in adult and aging rats, which are, presumably, not attributable to gonadal hormones. Further studies are needed to identify the potential genetic factors determining sex differences in DNase I activity in rats. Furthermore, the causal role of sex differences in DNase I and in some autoimmune diseases should be elucidated.

\section{$\mathrm{L}-27$}

\section{Variability in deoxyribonuclease activity and extracellular DNA in mice}

\section{Lubica Janovicova, Barbora Konecna, Jozef Conka, Lucia Laukova, Peter Celec}

Institute of Molecular Biomedicine, Faculty of Medicine, Comenius University, Bratislava, Slovak Republic

Correspondence to: lubica.janovicova@gmail.com

Deoxyribonucleases (DNases) can be secreted outside of cells and cleaveextracellular DNA (ecDNA). Exogenous DNase was shown to prevent mortality and tissue damage in animal models of sepsis, liver and kidney injury. We, therefore, hypothesize that endogenous plasma DNases could be important for ecDNA cleavage. The aim of our study was to explore biological variability of endogenous plasma DNase activityin conjunction with variability of ecDNA concentration. DNase activity was analyzed in plasma of healthy CD-1 mice $(n=72)$ using single radial enzyme diffusion assay. Concentration of ecDNA in plasma was determined using a fluorometric method and real-time PCR targeting both, nuclear and mitochondrial fractions of ecDNA.Sex difference in endogenous DNase activity was observed in mice, with males having 58\% higher DNase activity compared to females $(P 0.001)$. No associations between DNase activity and age or body weight of mice were found. The day-to-day variability in plasmatic DNase activity was very high with a coefficient of variation of $48 \%$ in female and $83 \%$ in male mice. On the other hand, sex differences were found neither in total ecDNA nor in nuclear or mitochondrial
ecDNA. Causes and consequences of biological variability of DNase activity and ecDNA should be investigated further, especially in humans, as DNase activity was shown to be much lower in rats and humans when compared to mice.

$\mathrm{L}-28$

\section{Novel miRNA downregulations in blastemal Wilms' tumor: An FFPE-based study}

\author{
Gergely Buglyo ${ }^{a}$, Zsofia Magyar ${ }^{b}$, Eva Romicsne Gorbe ${ }^{b}$, \\ Rita Banusz', Monika Csoka', Tamas Micsik ${ }^{\mathrm{d}}$, Zsanett Berki ${ }^{\mathrm{a}}$, \\ Peter Varga ${ }^{b}$, Zoltan Sapi ${ }^{d}$, Balint Nagy ${ }^{\mathrm{a}}$
}

\author{
${ }^{a}$ Department of Human Genetics, Faculty of Medicine, University \\ of Debrecen, Debrecen, Hungary \\ ${ }^{b} 1$ st Department of Obstetrics and Gynaecology, Semmelweis \\ University, Budapest, Hungary

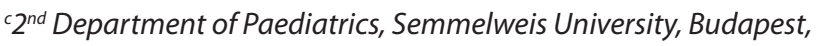 \\ Hungary \\ ${ }^{d} 7^{\text {st }}$ Department of Pathology and Experimental Cancer Research, \\ Semmelweis University, Budapest, Hungary
}

According to the SIOP protocol, Wilms' tumor histology is assessed from the surgical sample, after pre-operative chemotherapy. The two most common subtypes are regressive and blastemal. As the presence of blastema is a sign of adverse prognosis, recent efforts have focused on revealing molecular features of blastemal Wilms' tumors that may be responsible for poor chemo-responsiveness. Characteristic miRNA-signatures can be identified from tumor tissue as well as circulating miRNA from serum. In our study, we investigated miRNA expression in 8 patients using FFPE samples from tumor and control tissues. After identifying miRNAs of interest in the first sample by aqRT-PCR Array, 4 chosen miRNAs (miR-184, miR-203a, miR-34c-5p and miR-194-5p) were studied in all samples using individual primers. Results were comparable to previous reports based on fresh frozen tissue, but also showed a deregulation of miR-203a as a novel finding, and an underexpression of miR-184 at a previously unreported magnitude that may be present in a subset of blastemal or other Wilms' tumors. As a conclusion, we may note that further investigation of miRNA expression patterns in Wilms' tumor subtypes may be warranted, and FFPE samples (not older than 7-10 years) are excellent miRNA sources to increase sample count for high-scale studies. 


\section{$\mathrm{L}-29$}

\section{Causes and consequences of the variability of extracellular DNA}

\section{Peter Celec, Barbora VIkova}

Institute of Molecular Biomedicine, Faculty of Medicine, Comenius University, Bratislava, Slovak Republic

Correspondence to: petercelec@gmail.com

The analysis of cell-free, plasma or extracellular DNA (ecDNA) has many important diagnostic applications in oncology and prenatal medicine. The biology of ecDNA is everything but clear - not much is known about its origin, even less about its fate. For cells, however, ecDNA can be a damage-associated molecular pattern molecule - an inducer of inflammation. Numerous experiments have shown that inflammation is an important source of ecDNA, especially in form of extracellular traps formed by im- mune cells. The concentration of ecDNA in plasma rises during sepsis, but also after trauma and liver or kidney damage. In several published experiments using knockout mice it was proved that the recognition of ecDNA via TLR9 is crucial for its pro-inflammatory properties in various disease models. In addition, ecDNA cleavage induced by the administration of exogenous deoxyribonuclease reduces mortality in sepsis and morbidity in a model of hepatorenal failure. These reproducible findings together with the recently published proof of the importance of deoxyribonuclease 1 and 1-like 3 in the prevention of blood clots during neutrophilia lead to a provocative question: what is the role of the variability of the endogenous deoxyribonucleases in the risk of ecDNA-related diseases and their complications? Further research of this subject is definitely needed -not only to understand the biological basis of ecDNA cleavage, but also for direct application in clinical medicine where the low, but variable ecDNA concentration limits some of its diagnostic applications. 
$\mathrm{P}-1$

\section{Automation of cell-free DNA Testing on Ion Proton System}

\author{
Bozidara Hrindova, Iva Matejckova, Filip Zembol, \\ Martina Bittoova, Martin Hynek, Jana Vavrova, \\ Monika Koudova, David Stejskal
}

Centre of Medical Genetics and Reproductive Medicine, Gennet, Ltd., Kostelni 9, Prague 7, Czech Republic

Correspondence to: Bozidara.Hrindova@gennet.cz, Iva.Matejckova@gennet.cz

Introduction and Aim. Circulating cell-free DNA (cfDNA) in maternal plasma is used for highly efficient non-invasive prenatal screening for most common trisomis (NIPS). In this study our NIPS experience is presented, in which we use automated workflow of nucleic acid extraction and next-generation sequencing (NGS) library preparation. Our in-house NIPS method is based on standard low-coverage whole-genome NGS of cfDNA fragments, followed by customized bioinformatic analysis of relative frequency of reads aligned to corresponding chromosomes. The aim of the lecture is to compare advantages and disadvantages of manually and automatically cell-free DNA extraction and preparation of DNA libraries. Due to greater accuracy we introduced a gender verification method into our workflow. Automated laboratory workflow can reduce the risk of sample handling or cross contamination and increase the quality and uniformity of the DNA library.

Material and Methods. Protocols for cfDNA extraction from maternal plasma samples using the QIAsymphony AS (QIAGEN) automated system with QIAsymphony Circulating DNA kit and automated DNA library preparation using Sciclone G3 Liquid Handling Workstation (PerkinElmer) with Ion PlusFragment Library Kit (Life Technologies) were implemented.

Results and Discussion. The implementation of these two devices into routine laboratory practice greatly contributed to the acceleration of the whole methodology. Both devices are able to analyze up to 96 samples at a time. The resulting concentrations of samples measured on TapeStation 2200 (Agilent Technologies) are more uniform compared to manually prepared samples and the chance of human error introduction is significantly lower. Automated laboratory workflow can reduce risks of sample mishandling or cross contamination. The disadvantage of automation is a larger consumption of reagents and the associated higher cost of the analysis.

\section{P-2}

\section{Study the role of miR141 and miR429 in the diagnosis of epithelial ovarian cancer}

\author{
Eva Marton ${ }^{\mathrm{a}}$, Janos Lukacs ${ }^{\mathrm{b}}$, Reka Szabo a, Beata Soltesz ${ }^{\mathrm{a}}$, \\ Eszter Janka', Robert Pokab ${ }^{\mathrm{b}}$, Balint Nagy ${ }^{\mathrm{a}}$, \\ Melinda Szilagyi-Bonizs ${ }^{\mathrm{a}}$
}

\author{
${ }^{a}$ Department of Human Genetics, Faculty of Medicine, University \\ of Debrecen, Debrecen, Hungary \\ ${ }^{b}$ Department of Obstetrics and Gynecology, Faculty of Medicine, \\ University of Debrecen, Debrecen, Hungary \\ 'Department of Dermatology, Faculty of Medicine, University of \\ Debrecen, Debrecen, Hungary
}

Ovarian cancer is the most lethal form of gynecological malignancy that is mostly due to the difficulty of early diagnosis. Developing a fast, non-invasive diagnostic test would greatly facilitate the early detection and survival chances of ovarian cancer. Circulating miRNAs proved to be promising biomarkers in various cancers, including breast, prostate, pancreatic or colon cancer. However, only few publications focus on circulating miRNAs in ovarian cancer especially in European populations.

Here we studied the expression levels of miR141 and miR429 in the plasma samples of healthy $(\mathrm{n}=17)$, and previously untreated epithelial ovarian carcinoma patients ( $n=17$, FIGO stages III or IV) in a Hungarian cohort. The relative amount of miRNAs was detected by qRT-PCR.

The expression levels of miR141 and miR429 proved to be higher in the malignant samples compared to the healthy donors $(P<0.05)$. ROC-AUC proved to be good in the case of both $m i R 141(0.737,95 \% \mathrm{CI}=0.565-0.909)$ and $\operatorname{miR} 429(0.727,95 \% \mathrm{CI}=0.552-0.901)$. Sensitivity and negative predictive value were higher in the case of $m i R 429$ $(76.47 \%$ and $75 \%)$ than in the case of $m i R 141(64.71 \%$ and $68.42 \%$ ). However, specificity and positive predictive value proved to be higher in the case of $m i R 141(76.47 \%$ and $73.33 \%)$ than in the case of miR429 $(70.59 \%$ and $72.22 \%)$. Diagnostic accuracy was higher in the case of miR429 (73.53\%) than in the case of miR141 (70.59\%).

We conclude that the $m i R 200$ family member $m i R 141$ and miR429 might be promising candidate biomarkers in the diagnosis of ovarian cancer. Further studies are needed to test our hypothesis. 
$\mathrm{P}-3$

Determination of common chromosomal abnormalities with NIFTY Test (Non-invasive Fetal Trisomy Test) in Turkey

\author{
Ayse Sayli, Eren Akdeniz, Ibrahim Araz, Nurseda Kahveci, \\ Guzin Kokturk, Nagehan Ture, Merve Bektas, Gamze Serim, \\ Zeynep Bilgin, Dilek Ozdemir
}

\section{GENOKS Genetic Diagnostic Center, Ankara, Turkey \\ Correspondence to: erenakdeniz@genoks.com.tr}

Thanks to scientific developments, detection of many common chromosomal anomalies in the prenatal period has great importance on mother and baby health. Nextgeneration sequencing technology provides early detection and diagnosis in many medical situations. In this study, NIFTY Test (noninvasive fetal trisomy) based on Next Generation Sequencing technology is used to determine fetal chromosomal aneuploidies in the prenatal period. NIFTY Test is a kind of the NIPD (noninvasive prenatal diagnosis) tests which determine common trisomy abnormalities (T21, T13, T18) along with detection of 16 different chromosomal abnormalities in fetus chromosomes from cfDNA.

The test was performed by using $5-10 \mathrm{ml}$ peripheral blood samples from pregnant women. Wet-lab steps of the test are formed from plasma separation, DNA isolation, library preparation, and sequencing. This part of the test was performed in Turkey while sequencing data results were analyzed and reported by BGI (Shenzhen, China).

Between 2013-2018 (until April), NIFTY Test was studied on approximately 15.500 pregnant women samples aged 17-57 years in Turkey. 15.250 of the nifty test results were negative. High risk was detected in approximately 350 patients and suggested to be directed to invasive prenatal diagnosis, thus confirming the NIFTY test results. In addition to detection of fetal aneuploidies by NIFTY test, maternal cancer was detected in two of the patients. The studies and research for these two cases continue in communication with BGI and patients' physicians.

As a result of this study, valuable statistical data were obtained based on patients profiles and it has been indicated that prefer ability of NIFTY test has been increasing according to other biochemical-conventional tests and competing NIPD tests in Turkey and near countries because of its high accuracy, sensitivity and specificity ratio.
P-4

\section{Determination of PITX2 and NEURL1 single nucleotide polymorphisms in atrial fibrillation}

\author{
Krisztina Szirak ${ }^{\mathrm{a}}$, Beata Soltesz ${ }^{\mathrm{a}}$, Orsolya Hajas ${ }^{\mathrm{b}}$, \\ Reka Urbancsek ${ }^{b}$, Edina Nagy-Balo ${ }^{\mathrm{b}}$, Andras Penyige ${ }^{\mathrm{a}}$, \\ Zoltan Csanadi ${ }^{b}$, Balint Nagy ${ }^{a}$
}

a Departmant of Human Genetics, Faculty of Medicine, University of Debrecen, Debrecen, Hungary

'Institute of Cardiology, Faculty of Medicine, University of Debrecen, Debrecen, Hungary

Introduction. Atrial fibrillation (AF) is the most common cardiac arrhythmia affecting $1-2 \%$ of the population. PITX2 and NEURL1 SNP polymorphism was described as associated with AF. PITX2 is a homeobox transcription factor that plays a pivotal role during embryonic development. Independent genome-wide association studies reported several risk variants such as rs 2595104 close to the PITX2 locus on chromosome 4q25 that are strongly associated with AF in humans. Also NEURL1 is an E3 ubiquitin ligase involved in internalization and degradation of Notch ligands in signal-sending cells, which is required to activate Notch signaling. NEURL1 rs6584555 correlated with an increased risk to AF in previous studies.

Materials and Methods. We involved 56 atrial fibrillation patients and 58 controls in this study. 15-16 ml EDTA blood was drawn and we isolated the DNA with silica adsorption method. We used LightSnip kit (TibMolbiol, Berlin, Germany) for determination of rs2595104 and rs6584555 polymorphisms. We determined the melting temperatures of the PCR fragments by using LightCycler 96 instrument and calculated the allele, and genotype frequencies, and used chi-squared test for the statistical calculations.

Results. The frequency of A risk allele in the case of PITX2 was 0.41 in the control group and 0.44 in the AF patients. The log additive odds ratio is 1.142 ; C.I. $=0.675$ 1.932; $P=0.621$.The frequency of $\mathrm{C}$ risk allele in the case of NEURL1was 0.23 in the control group and 0.25 in the AF patients. The log additive odds ratio is 1,116; C.I. $=0.613-2.032 ; P=0.718$.

Conclusions. We determined the rs2595104 and rs6584555SNP polymorphisms in group of Hungarian atrial fibrillation patients, while we did not find significant differences in the genotype frequencies. We made a pilot study on the determination of PITX2 SNP polymorphism from cell-free DNA isolated from exosomes. 
P-5

Comparative analysis of the somatic mutation profiles in monitored 26 genes in circulating tumor DNA from breast cancer patients before, after surgery and after adjuvant chemotherapy the pilot study

\author{
Katarina Zelinova ${ }^{\mathrm{a}, \mathrm{b}}$, Marianna Jagelkova ${ }^{\mathrm{a}, \mathrm{b}}$, \\ Zuzana Laucekova ${ }^{\mathrm{b}}$, Zuzana Dankova ${ }^{\mathrm{a}}$, Marian Grendarc, \\ Karol Dokus ${ }^{\mathrm{b}, \mathrm{d}}$
}

${ }^{a}$ Department of Oncology, Biomedical Center Martin, Jessenius Faculty of Medicine in Martin, Comenius University in Bratislava (JFM CU), 03601 Martin, Slovak Republic

${ }^{b}$ Department of Obstetrics and Gynecology, Martin University Hospital and JFM CU, 03601 Martin, Slovak Republic

'Department of Bioinformatics, Biomedical Center Martin JFM CU, 03601 Martin, Slovak Republic

${ }^{d} 2^{\text {nd }}$ Department of Obstertrics and Gynecology, Slovak Medical University, Faculty Hospital with Polyclinic of F. D. Roosevelt, 975 17 Banska Bystrica, Slovak Republic

Correspondence to: marianna.jagelkova@uniba.sk

Introduction. Breast cancer is the most common diagnosed oncological genetic disease in women. Circulating tumour DNA released from apoptotic and necrotic tumour cells contains complete information about genetic changes of tumour and can be analysed by next-generation sequencing.

Methods. Blood samples were collected into EDTA tubes from patients with breast carcinoma at three time points: before, after surgery and after adjuvant chemotherapy. CtDNA was isolated from plasma by QIAamp DSP Virus kit (QIAGEN, Germany) and was concentrated by vacuum system (LABCONCO, USA). DNA libraries were prepared by TruSight Tumor 26 kit and their quality and quantity were measured by Qubit 2.0 Fluorometer (Invitrogen, USA) and 2100 Bioanalyzer (Agilent Technologies, Germany). The bioinformatic analysis of sequencing data was performed through Variant Effect Predictor, VarSome and COSMIC (v84).
Results. We analysed somatic gene profile of four patients and detected several variants in 11 genes such as PTEN, KRAS, TP53, APC, KIT, MET, PDGFRA, BRAF, CTNNB1, PIK3CA, FBXW7. In samples before surgery, we identified $5 / 10$ variants (patient 1 ), 5/7 variants (patient 2), 3/5 variants (patient 3), 2/5 variants (patient 4 ), which were confirmed as somatic pathogenic and were not detected in samples after surgery and chemotherapy (tab. 1).

Conclusion. Comparison of the gene profile showed that somatic pathogenic variants were not present in ctDNA samples as early as after surgery.

Acknowledgement: This work was supported by The Slovak Research and Development Agency with number 14-0815.

Table 1. Identified somatic pathogenic variants in samples before surgery.

\begin{tabular}{cll}
\hline Gene & Variant & Effect \\
\hline Patient 1 & & \\
PTEN & c.43A $>\mathrm{G} ;$ & missense \\
& c.101C $>\mathrm{A}$ & \\
KRAS & c.350A $>\mathrm{G}$ & $\begin{array}{l}\text { missense } \\
\text { missense }\end{array}$ \\
AP53 & c.376T $>\mathrm{C}$ & nonsense/stop gain \\
\hline Patient 2 & c.4495G $>\mathrm{T}$ & \\
CTNNB1 & c.101G $>\mathrm{A} ;$ & missense \\
& c.121A $>\mathrm{C}$ & \\
PIK3CA & c.3139C $>\mathrm{T}$ & missense \\
FBXW7 & c.1123G $>\mathrm{A}$ & missense \\
BRAF & c.1799T $>\mathrm{A}$ & missense \\
\hline Patient 3 & & \\
PTEN & c.210-2A $>\mathrm{G}$ & splice acceptor variant \\
TP53 & c.293C $>\mathrm{T}$ & missense \\
APC & c.3373G $>\mathrm{C}$ & missense \\
\hline Patient 4 & & \\
TP53 & c.1031T $>\mathrm{C}$ & missense \\
PIK3CA & c.29T $>\mathrm{C}$ & missense \\
\hline
\end{tabular}


CP-1

\section{NIFTY-A reliable NIPT technology}

\section{Zhou Yafeng}

\section{BGI International Dx Unit}

Since BGI launched the first commercial NGS-based NIPT test, it has been well recognized and incorporated into the public health systems in many countries including US, UK, etc. It is also recommended as the first line screening method forthe huge advantages in accuracy and completeness comparing to the traditional serum screening method. As the world's first and largest NIPT service provider, BGI has completed over 3 million tests worldwide in more than 70 countries. Its NIPT test, namely NIFTY, now detects trisomy of 6 chromosomes, sex chromosome aneuploidies and micro deletion and duplication related to 84 inherited diseases. BGI is also building V-labs all over the world with its local partners to provide better services.

\section{CP-2}

\section{Sentis- helpful tool for tumor screening}

\section{Zhou Yafeng}

BGI International Dx Unit

Beside fetal DNA, another important source of cell free DNA is circulating tumor DNA (ctDNA). The detection of ctDNA is useful in the early detection, subtype classification, and relapse surveillance of cancer. BGI also provides various sequencing products in precision oncology, including hereditary cancer screening, companion diagnosis for targeted drug or immunotherapy, discovery of new biomarkers, etc. 


\section{AUTHOR INDEX}

Adleff V. L-16

Akdeniz E. P-3

Aksu G. L-21

Anagnostou V. L-16

Araz I. P-3

Asselbergs F. IS-5

Balaz A. L-25

Balla B. L-2

Bancos I. L-13

Banusz R. L-28

Bedekovics J. L-14

Bektas M. P-3

Berki Z. L-28

Bilgin Z. P-3

Biro O. L-9; L-23; L-25

Bittoova M, P-1

Bodor C. L-11; L-15

Boscaro M. L-13

Brahmer RJ. L-16

Bruhm CD. L-16

Budis J. L-25

Buglyo G, L-28

Burjanivova T. L-17

Butz H. L-13

Buzas IE. L-13

Cabuk D. L-21

Celec P. L-26; L-27; L-29

Christensen R. IS-4

Christesen BI. IS-4

Cine N. L-21

Conka J. L-27

Cristiano S. L-16

Csanadi Z. L-7; P-4

Csaszar K. L-15

Csoka M, L-28

Cuckle H. IS-1; IS-3

Danko J. L-1

Dankova Z. P-5

Decmann A. L-13

Demir G. L-21

Dokus K. P-5

Domonkos E, L-26

Duygu Y. L-21

Egedi K. L-15

Fiksel J. L-16

Forde MP. L-16

Gazdaricova I. L-25

Gok I. L-22

Gokbayrak M. L-21

Gorenjec NR. L-3

Grendar M. L-1; L-17; P-5

Guzdolu E. L-21

Hajas O. P-4

Hatt L. IS-4

Hopfner T. L-26
Hornakova A. L-1

Hrindova B. P-1

Hromadnikova I. L-5; L-6

Hron F. L-6

Hrtankova M. L-1

Husain H. L-16

Igaz P. L-10, L-13

Isik U. L-21

Ivankova K. L-5

Jagelkova M. P-5

Janka E. L-19; P-2

Janovicova L. L-26; L-27

Josipovic G. L-24

Kahveci N. P-3

Kajsik M. L-25

Keseru J. L-18

Keszne Fodor L. L-2

Klujber V. L-2

Kokturk G. P-3

Konecna B. L-27

Kosa J. L-2

Kotlabova K. L-5; L-6

Koudova M. P-1

Kovalszky I. L-11; L-15

Kovesdi A. L-2

Krofta L. L-5; L-6

Lakatos P. L-2

Lasabova Z. L-1; L-17

Laucekova Z. L-1; P-5

Laukova L. L-27

Leal A. L-16

Loderer D. L-1

Luconi M. L-13;

Lukacs J. L-18; L-19; L-20; L-23; P-2

Magyar Z. L-28

Malicherova B. L-17

Mannelli M. L-13

Marrone AK. L-16

Marton E. L-19; P-2

Matejckova I. P-1

Mehes G. L-14

Micsik T. L-28

Mokanszki A. L-14

Molnar G. L-2

Nagy B, L-1; L-8; L-9; L-18; L-19; L-20; L-23; L-25; L-28; P-2; P-4

Nagy-Balo E. P-4

Olah L. L-15

Ozdemir D. P-3

Ozden E. L-21

Ozden O. L-22

Papp G. L-11

Patocs A. L-13

Penyige A.L-19; L-23; P-4
Perge P. L-13

Petersen BO. IS-4

Pezzani R. L-13

Phallen J. L-16

Plank L. L-17

Poka R. L-12; L-18; L-19; L-20; L-23; P-2

Ravn K. IS-4

Rigo J. L-9

Romicsne Gorbe E. L-28

Sapi Z. L-28

Savli H. L-21

Sayli A. P-3

Scharpf BR. L-16

Schelde P. IS-4

Sensen C. IS-6

Serim G. P-3

Sertdemir N. L-21

Singh R. IS-4

Sistermans E. IS-2

Soltesz B. L-18; L-19; L-20; L-23; P-2; P-4

Stehle T. L-26

Steinhardt M. L-26

Stejskal D. P-1

Strah D. L-3

Svecova I. L-1

Szabo R. P-2

Szemes T. L-25

Szilagyi E. L-19

Szilagyi-Bonizs M. L-19; P-2

Szirak K. P-4

Szoke S. L-2

Tadic V. L-24

Tobias B. L-2

Toth M. L-13

Torok O. L-4

Ture N. P-3

Turna J. L-25

Uldbjerg N. IS-4

Urbancsek R. P-4

Uygun K. L-21

Vanova B. L-17

Varga P. L-28

Velculescu EV. L-16

Vlkova B. L-29

Vogel I. IS-4

Vojta A. L-24

Weihe E. L-16

Woodward B. L-16

Zelinova K. P-5

Zembol F. P-1

Zoldos V. L-24

Zhou Y. CP-1; CP-2

Yavuz D. L-21 
SPONSORS
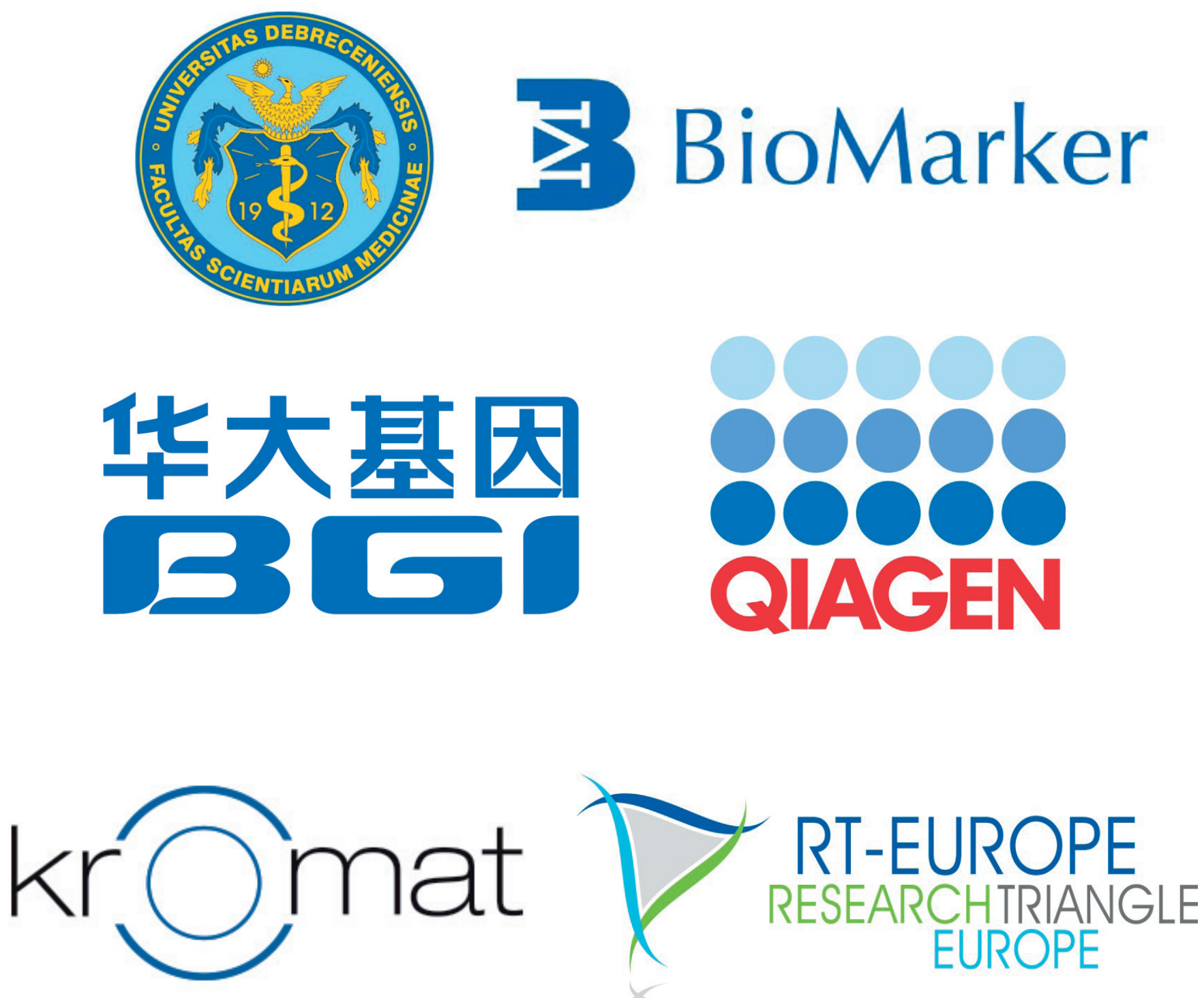

Agilent Technologies

—_Authorized Distributor

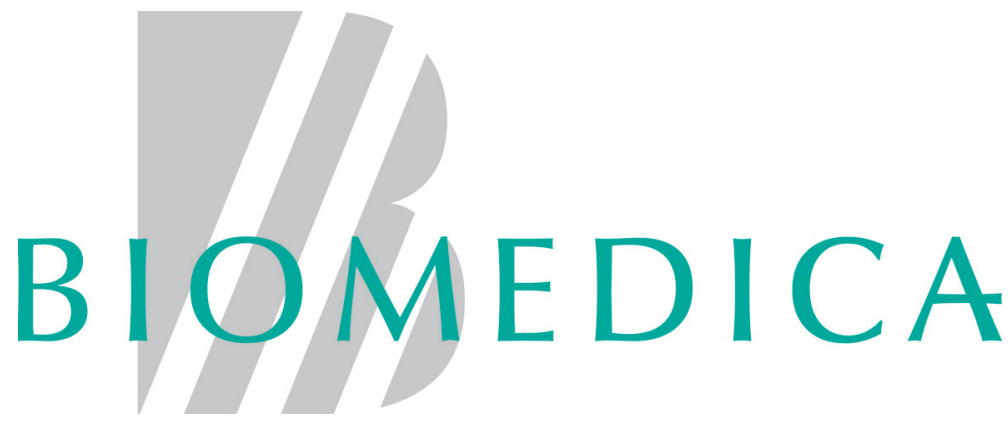

